\title{
Schur Product with Operator-valued Entries
}

\author{
Oscar Blasco and Ismael García-Bayona*
}

\begin{abstract}
In this paper we characterize Toeplitz matrices with entries in the space of bounded operators on Hilbert spaces $\mathcal{B}(H)$ which define bounded operators acting on $\ell^{2}(H)$ and use it to get the description of the right Schur multipliers acting on $\ell^{2}(H)$ in terms of certain operator-valued measures.
\end{abstract}

\section{Introduction}

Throughout the paper $X, Y$ and $E$ are complex Banach spaces and $H$ denotes a separable complex Hilbert space with orthonormal basis $\left(e_{n}\right)$. We write $\mathcal{L}(X, Y)$ for the space of bounded linear operators, $X^{*}$ for the dual space and denote $\mathcal{B}(X)=\mathcal{L}(X, X)$. We also use the notations $\ell^{2}(E), C(\mathbb{T}, E), L^{p}(\mathbb{T}, E)$ or $M(\mathbb{T}, E)$ for the space of sequences $\mathbf{z}=\left(z_{n}\right)$ in $E$ such that $\|\mathbf{z}\|_{\ell^{2}(E)}=\left(\sum_{n=1}^{\infty}\left\|z_{n}\right\|^{2}\right)^{1 / 2}<\infty$, the space of $E$-valued continuous functions, the space of strongly measurable functions from the measure space $\mathbb{T}=\{z \in \mathbb{C}:|z|=1\}$ into $E$ with $\|f\|_{L^{p}(\mathbb{T}, E)}=\left(\int_{0}^{2 \pi}\left\|f\left(e^{i t}\right)\right\|^{p} \frac{d t}{2 \pi}\right)^{1 / p}<\infty$ for $1 \leq p \leq \infty$ (with the usual modification for $p=\infty$ ) and the space of regular vector-valued measures of bounded variation respectively. As usual, for $E=\mathbb{C}$ we simply write $\ell^{2}, C(\mathbb{T}), L^{p}(\mathbb{T})$ and $M(\mathbb{T})$.

Given two matrices $A=\left(\alpha_{k j}\right)$ and $B=\left(\beta_{k j}\right)$ with complex entries, their Schur product is defined by $A * B=\left(\alpha_{k j} \beta_{k j}\right)$. This operation endows the space $\mathcal{B}\left(\ell^{2}\right)$ with a structure of Banach algebra. A proof of the next result, due to J. Schur, can be found in 2 , Proposition 2.1] or [10, Theorem 2.20].

Theorem 1.1. (Schur, 12]) If $A=\left(\alpha_{k j}\right) \in \mathcal{B}\left(\ell^{2}\right)$ and $B=\left(\beta_{k j}\right) \in \mathcal{B}\left(\ell^{2}\right)$ then $A * B \in$ $\mathcal{B}\left(\ell^{2}\right)$. Moreover $\|A * B\|_{\mathcal{B}\left(\ell^{2}\right)} \leq\|A\|_{\mathcal{B}\left(\ell^{2}\right)}\|B\|_{\mathcal{B}\left(\ell^{2}\right)}$.

More generally, a matrix $A=\left(\alpha_{k j}\right)$ is said to be a Schur multiplier, to be denoted by $A \in \mathcal{M}\left(\ell^{2}\right)$, whenever $A * B \in \mathcal{B}\left(\ell^{2}\right)$ for any $B \in \mathcal{B}\left(\ell^{2}\right)$. For the study of Schur multipliers we refer the reader to 2,10$]$. Recall that a Toeplitz matrix is a matrix $A=\left(\alpha_{k j}\right)$ such that there exists a sequence of complex numbers $\left(\gamma_{l}\right)_{l \in \mathbb{Z}}$ with $\alpha_{k j}=\gamma_{j-k}$. The study of

Received December 21, 2017; Accepted November 18, 2018.

Communicated by Xiang Fang.

2010 Mathematics Subject Classification. Primary: 46E40; Secondary: 47A56, 15 B05.

Key words and phrases. Schur product, Toeplitz matrix, Schur multiplier, vector-valued measures.

This work was partially supported by MTM2014-53009-P (MINECO Spain) and FPU14/01032 (MCIU Spain).

*Corresponding author. 
Toeplitz matrices which define bounded operators or Schur multipliers goes back to work of Toeplitz in [15]. The reader is referred to [1,2,10] for recent proofs of the following results concerning Toeplitz matrices.

Theorem 1.2. (Toeplitz, 15$)$ Let $A=\left(\alpha_{k j}\right)$ be a Toeplitz matrix. Then $A \in \mathcal{B}\left(\ell^{2}\right)$ if and only if there exists $f \in L^{\infty}(\mathbb{T})$ such that $\alpha_{k j}=\widehat{f}(j-k)$ for all $k, j \in \mathbb{N}$. Moreover $\|A\|=\|f\|_{L^{\infty}(\mathbb{T})}$.

Theorem 1.3. (Bennet, 2]) Let $A=\left(\alpha_{k j}\right)$ be a Toeplitz matrix. Then $A \in \mathcal{M}\left(\ell^{2}\right)$ if and only if there exists $\mu \in M(\mathbb{T})$ such that $\alpha_{k j}=\widehat{\mu}(j-k)$ for all $k, j \in \mathbb{N}$. Moreover $\|A\|=\|\mu\|_{M(\mathbb{T})}$.

It is known the recent interest for operator-valued functions (see [9]) and for the matricial analysis (see [10]) concerning their uses in different problems in Analysis. In this paper, we would like to formulate the analogues of the theorems above in the context of matrices $\mathbf{A}=\left(T_{k j}\right)$ with entries $T_{k j} \in \mathcal{B}(H)$. For such a purpose, we are led to consider operator-valued measures. We shall make use of several notions and spaces from the theory of vector-valued measures and the reader is referred to classical books [6, 7] or to [3] for some new results in connection with Fourier analysis.

In the sequel we write $\langle\cdot, \cdot\rangle$ and $\langle\langle\cdot, \cdot\rangle\rangle$ for the scalar products in $H$ and $\ell^{2}(H)$ respectively, where $\langle\langle\mathbf{x}, \mathbf{y}\rangle\rangle=\sum_{j=1}^{\infty}\left\langle x_{j}, y_{j}\right\rangle$ and we use the notation $x \mathbf{e}_{j}=(0, \ldots, 0, x, 0, \ldots)$ for the element in $\ell^{2}(H)$ in which $x \in H$ is placed in the $j$-coordinate for $j \in \mathbb{N}$. As usual, $c_{00}(H)=\operatorname{span}\left\{x \mathbf{e}_{j}: x \in H, j \in \mathbb{N}\right\}$.

Definition 1.4. Given a matrix $\mathbf{A}=\left(T_{k j}\right)$ with entries $T_{k j} \in \mathcal{B}(H)$ and $\mathbf{x} \in c_{00}(H)$ we write $\mathbf{A}(\mathbf{x})$ for the sequence $\left(\sum_{j=1}^{\infty} T_{k j}\left(x_{j}\right)\right)_{k}$. We say that $\mathbf{A} \in \mathcal{B}\left(\ell^{2}(H)\right)$ if the map $\mathbf{x} \rightarrow \mathbf{A}(\mathbf{x})$ extends to a bounded linear operator in $\ell^{2}(H)$, that is

$$
\left(\sum_{k=1}^{\infty}\left\|\sum_{j=1}^{\infty} T_{k j}\left(x_{j}\right)\right\|^{2}\right)^{1 / 2} \leq C\left(\sum_{j=1}^{\infty}\left\|x_{j}\right\|^{2}\right)^{1 / 2}
$$

We shall write

$$
\|\mathbf{A}\|_{\mathcal{B}\left(\ell^{2}(H)\right)}=\inf \left\{C \geq 0:\|\mathbf{A} \mathbf{x}\|_{\ell^{2}(H)} \leq C\|\mathbf{x}\|_{\ell^{2}(H)}\right\}
$$

Definition 1.5. Given two matrices $\mathbf{A}=\left(T_{k j}\right)$ and $\mathbf{B}=\left(S_{k j}\right)$ with entries $T_{k j}, S_{k j} \in$ $\mathcal{B}(H)$ we define the Schur product $\mathbf{A} * \mathbf{B}=\left(T_{k j} S_{k j}\right)$ where $T_{k j} S_{k j}$ stands for the composition of the operators $T_{k j}$ and $S_{k j}$.

Contrary to the scalar-valued case this product is not commutative. 
Definition 1.6. Given a matrix $\mathbf{A}=\left(T_{k j}\right)$, we say that $\mathbf{A}$ is a right Schur multiplier (respectively left Schur multiplier), to be denoted by $\mathbf{A} \in \mathcal{M}_{r}\left(\ell^{2}(H)\right.$ ) (respectively $\mathbf{A} \in$ $\left.\mathcal{M}_{l}\left(\ell^{2}(H)\right)\right)$, whenever $\mathbf{B} * \mathbf{A} \in \mathcal{B}\left(\ell^{2}(H)\right)$ (respectively $\mathbf{A} * \mathbf{B} \in \mathcal{B}\left(\ell^{2}(H)\right)$ ) for any $\mathbf{B} \in$ $\mathcal{B}\left(\ell^{2}(H)\right)$. We shall write

$$
\|\mathbf{A}\|_{\mathcal{M}_{r}\left(\ell^{2}(H)\right)}=\inf \left\{C \geq 0:\|\mathbf{B} * \mathbf{A}\|_{\mathcal{B}\left(\ell^{2}(H)\right)} \leq C\|\mathbf{B}\|_{\mathcal{B}\left(\ell^{2}(H)\right)}\right\}
$$

and

$$
\|\mathbf{A}\|_{\mathcal{M}_{l}\left(\ell^{2}(H)\right)}=\inf \left\{C \geq 0:\|\mathbf{A} * \mathbf{B}\|_{\mathcal{B}\left(\ell^{2}(H)\right)} \leq C\|\mathbf{B}\|_{\mathcal{B}\left(\ell^{2}(H)\right)}\right\} .
$$

Denoting by $\mathbf{A}^{*}$ the adjoint matrix given by $S_{k j}=T_{j k}^{*}$ for all $k, j \in \mathbb{N}$, one easily sees that $\mathbf{A} \in \mathcal{B}\left(\ell^{2}(H)\right)$ if and only if $\mathbf{A}^{*} \in \mathcal{B}\left(\ell^{2}(H)\right)$ with $\|\mathbf{A}\|=\left\|\mathbf{A}^{*}\right\|$ and also that $\mathbf{A} \in \mathcal{M}_{l}\left(\ell^{2}(H)\right)$ if and only if $\mathbf{A}^{*} \in \mathcal{M}_{r}\left(\ell^{2}(H)\right)$ and $\|\mathbf{A}\|_{\mathcal{M}_{l}\left(\ell^{2}(H)\right)}=\left\|\mathbf{A}^{*}\right\|_{\mathcal{M}_{r}\left(\ell^{2}(H)\right)}$.

If $X$ and $Y$ are Banach spaces we write $X \widehat{\otimes} Y$ for the projective tensor product. We refer the reader to [6, Chapter 8], [11, Chapter 2] or [4] for all possible results needed in the paper. We recall that $(X \widehat{\otimes} Y)^{*}=\mathcal{L}\left(X, Y^{*}\right)$ and to avoid misunderstandings, for each $T \in \mathcal{L}\left(X, Y^{*}\right)$, we write $\mathcal{J} T$ when $T$ is seen as an element in $(X \widehat{\otimes} Y)^{*}$. In other words, we write $\mathcal{J}: \mathcal{L}\left(X, Y^{*}\right) \rightarrow(X \widehat{\otimes} Y)^{*}$ for the isometry given by $\mathcal{J} T(x \otimes y)=T(x)(y)$ for any $T \in \mathcal{L}\left(X, Y^{*}\right), x \in X$ and $y \in Y$. Also, given $x^{*} \in X^{*}$ and $y^{*} \in Y^{*}$, we write $\widetilde{x^{*} \otimes y^{*}}$ for the operator in $\mathcal{L}\left(X, Y^{*}\right)$ given by $\widetilde{x^{*} \otimes y^{*}}(z)=x^{*}(z) y^{*}$ for each $z \in X$. In the paper we shall restrict ourselves to the case $\mathcal{L}\left(X, Y^{*}\right)=\mathcal{B}(H)$, that is $X=Y^{*}=H$. Using the Riesz theorem we identify $Y=Y^{*}=H$. Hence, for $T, S \in \mathcal{B}(H)$ and $x, y \in H$, we shall use the following formulae

$$
\begin{gathered}
\langle T(x), y\rangle=\mathcal{J} T(x \otimes y), \\
\widetilde{(x \otimes y)}(z)=\langle z, x\rangle y, \quad z \in H, \\
T \widetilde{x \otimes y})=(\widetilde{x \otimes(T y})), \quad \widetilde{(x \otimes y}) T=\left(\widetilde{\left.T^{*} x\right) \otimes y},\right. \\
\mathcal{J}(T S)(x \otimes y)=\mathcal{J} T(S x \otimes y)=\mathcal{J} S\left(x \otimes T^{*} y\right) .
\end{gathered}
$$

The paper is divided into four sections. The first section is of a preliminary character and we recall the basic notions on vector-valued sequences and functions to be used in the sequel. Next section contains several results on regular operator-valued measures which are the main ingredients for the remaining proofs in the paper. In Section 4 we are concerned with several necessary and sufficient conditions for a matrix $\mathbf{A}$ to belong to $\mathcal{B}\left(\ell^{2}(H)\right)$ and we show that the Schur product endows $\mathcal{B}\left(\ell^{2}(H)\right)$ with a Banach algebra structure also in this case. The final section deals with Toeplitz matrices $\mathbf{A}$ with entries in $\mathcal{B}(H)$, that is those matrices for which there exists a sequence $\left(T_{l}\right)_{l \in \mathbb{Z}} \subset \mathcal{B}(H)$ so that $T_{k j}=T_{j-k}$. We shall write $\mathcal{T}$ the family of such Toeplitz matrices and we characterize $\mathcal{T} \cap \mathcal{B}\left(\ell^{2}(H)\right)$ as those matrices where $T_{k j}=\widehat{\mu}(j-k)$ for a certain regular operator-valued vector measure 
$\mu$ belonging to $V^{\infty}(\mathbb{T}, \mathcal{B}(H)$ ) (see Definition 3.6 below). Concerning the analogue of Theorem 1.3 we shall show that $M(\mathbb{T}, \mathcal{B}(H)) \subseteq \mathcal{M}_{r}\left(\ell^{2}(H)\right) \subseteq M_{\mathrm{SOT}}(\mathbb{T}, \mathcal{B}(H))$ where $M(\mathbb{T}, \mathcal{B}(H))$ stands for the space of regular operator-valued measures and $M_{\mathrm{SOT}}(\mathbb{T}, \mathcal{B}(H))$ is defined, using the strong operator topology, as the space of vector measures $\mu$ such that $\mu_{x} \in M(\mathbb{T}, H)$ where $\mu_{x}(A)=\mu(A)(x)$ for any $x \in H$.

\section{Preliminaries on operator-valued sequences and functions}

Write $\ell_{\text {weak }}^{2}(\mathbb{N}, \mathcal{B}(H))$ and $\ell_{\text {weak }}^{2}\left(\mathbb{N}^{2}, \mathcal{B}(H)\right)$ for the space of sequences $\mathbf{T}=\left(T_{n}\right) \subset \mathcal{B}(H)$ and matrices $\mathbf{A}=\left(T_{k j}\right) \subset \mathcal{B}(H)$ such that

$$
\|\mathbf{T}\|_{\ell_{\text {weak }}^{2}(\mathbb{N}, \mathcal{B}(H))}=\sup _{\|x\|=1,\|y\|=1}\left(\sum_{n=1}^{\infty}\left|\left\langle T_{n}(x), y\right\rangle\right|^{2}\right)^{1 / 2}<\infty
$$

and

$$
\|\mathbf{A}\|_{\ell_{\text {weak }}^{2}\left(\mathbb{N}^{2}, \mathcal{B}(H)\right)}=\sup _{\|x\|=1,\|y\|=1}\left(\sum_{k=1}^{\infty} \sum_{j=1}^{\infty}\left|\left\langle T_{k j}(x), y\right\rangle\right|^{2}\right)^{1 / 2}<\infty .
$$

The reader can see that these spaces actually coincide with the ones appearing using notation in $[5]$. Of course $\ell^{2}(E) \subsetneq \ell_{\text {weak }}^{2}(E)$. In the case $\mathcal{B}(H)$ we can actually introduce certain spaces between $\ell^{2}(E)$ and $\ell_{\text {weak }}^{2}(E)$.

Definition 2.1. Given a sequence $\mathbf{T}=\left(T_{n}\right)$ and a matrix $\mathbf{A}=\left(T_{k j}\right)$ of operators in $\mathcal{B}(H)$, we write

$$
\|\mathbf{T}\|_{\ell_{\mathrm{SOT}}^{2}(\mathbb{N}, \mathcal{B}(H))}=\sup _{\|x\|=1}\left(\sum_{n=1}^{\infty}\left\|T_{n}(x)\right\|^{2}\right)^{1 / 2}
$$

and

$$
\|\mathbf{A}\|_{\ell_{\text {SOT }}^{2}\left(\mathbb{N}^{2}, \mathcal{B}(H)\right)}=\sup _{\|x\|=1}\left(\sum_{j=1}^{\infty} \sum_{k=1}^{\infty}\left\|T_{k j}(x)\right\|^{2}\right)^{1 / 2} .
$$

We set $\ell_{\mathrm{SOT}}^{2}(\mathbb{N}, \mathcal{B}(H))$ and $\ell_{\mathrm{SOT}}^{2}\left(\mathbb{N}^{2}, \mathcal{B}(H)\right)$ for the spaces of sequences and operators with $\|\mathbf{T}\|_{\ell_{\mathrm{SOT}}^{2}(\mathbb{N}, \mathcal{B}(H))}<\infty$ and $\|\mathbf{A}\|_{\ell_{\mathrm{SOT}}^{2}\left(\mathbb{N}^{2}, \mathcal{B}(H)\right)}<\infty$ respectively.

Remark 2.2. It is easy to show that

$$
\ell^{2}\left(\mathbb{N}^{2}, \mathcal{B}(H)\right) \subsetneq \ell^{2}\left(\mathbb{N}, \ell_{\mathrm{SOT}}^{2}(\mathbb{N}, \mathcal{B}(H)) \subsetneq \ell_{\mathrm{SOT}}^{2}\left(\mathbb{N}^{2}, \mathcal{B}(H)\right)\right.
$$

As usual, we denote $\varphi_{k}(t)=e^{i k t}$ for $k \in \mathbb{Z}$, and, given a complex Banach space $E$, we write $\mathcal{P}(\mathbb{T}, E)=\operatorname{span}\left\{e \varphi_{j}: j \in \mathbb{Z}, e \in E\right\}$ for the $E$-valued trigonometric polynomials, $\mathcal{P}_{a}(\mathbb{T}, E)=\operatorname{span}\left\{e \varphi_{j}: j \in \mathbb{N}, e \in E\right\}$ for the $E$-valued analytic polynomials. It is well-known that $\mathcal{P}(\mathbb{T}, E)$ is dense in $C(\mathbb{T}, E)$ and $L^{p}(\mathbb{T}, E)$ for $1 \leq p<\infty$. Also, we 
shall use $H_{0}^{2}(\mathbb{T}, E)=\left\{f \in L^{2}(\mathbb{T}, E): \widehat{f}(k)=0, k \leq 0\right\}$, where $\widehat{f}(k)=\int_{0}^{2 \pi} f(t) \overline{\varphi_{k}(t)} \frac{d t}{2 \pi}$ for $k \in \mathbb{Z}$. Recall that $H_{0}^{2}(\mathbb{T}, E)$ coincides with the closure of $\mathcal{P}_{a}(\mathbb{T}, E)$ with the norm in $L^{2}(\mathbb{T}, E)$. Similarly $H_{0}^{2}\left(\mathbb{T}^{2}, E\right)=\left\{f \in L^{2}\left(\mathbb{T}^{2}, E\right): \widehat{f}(k, j)=0, k, j \leq 0\right\}$, where $\widehat{f}(k, j)=\int_{0}^{2 \pi} \int_{0}^{2 \pi} f(t, s) \overline{\varphi_{k}(t) \varphi_{j}(s)} \frac{d t}{2 \pi} \frac{d s}{2 \pi}$ for $k, j \in \mathbb{Z}$.

Let us now introduce some new spaces that we shall need later on.

Definition 2.3. Let $\mathbf{T}=\left(T_{n}\right) \subset \mathcal{B}(H)$ and $\mathbf{A}=\left(T_{k j}\right) \subset \mathcal{B}(H)$. We say that $\mathbf{T} \in$ $\widetilde{H}^{2}(\mathbb{T}, \mathcal{B}(H))$ whenever

$$
\|\mathbf{T}\|_{\widetilde{H}^{2}(\mathbb{T}, \mathcal{B}(H))}=\sup _{N}\left(\int_{0}^{2 \pi}\left\|\sum_{j=1}^{N} T_{j} \varphi_{j}(t)\right\|^{2} \frac{d t}{2 \pi}\right)^{1 / 2}<\infty .
$$

We say that $\mathbf{A} \in \widetilde{H}^{2}\left(\mathbb{T}^{2}, \mathcal{B}(H)\right)$ whenever

$$
\|\mathbf{A}\|_{\widetilde{H}^{2}\left(\mathbb{T}^{2}, \mathcal{B}(H)\right)}=\sup _{N, M}\left(\int_{0}^{2 \pi} \int_{0}^{2 \pi}\left\|\sum_{j=1}^{N} \sum_{k=1}^{M} T_{k j} \varphi_{j}(t) \varphi_{k}(s)\right\|^{2} \frac{d t}{2 \pi} \frac{d s}{2 \pi}\right)^{1 / 2}<\infty .
$$

Remark 2.4. $\widetilde{H}^{2}(\mathbb{T}, \mathcal{B}(H)) \nsubseteq H_{0}^{2}(\mathbb{T}, \mathcal{B}(H))$.

Consider $T_{j}=\widehat{e_{j} \otimes e_{j}}$. Then for any $t \in[0,2 \pi)$ and $N \in \mathbb{N}$,

$$
\left.\| \sum_{j=1}^{N} \widetilde{\left(e_{j} \otimes e_{j}\right.}\right) \varphi_{j}(t)\left\|_{\mathcal{B}(H)}=\sup _{\|x\|=1}\right\| \sum_{j=1}^{N}\left\langle x, e_{j}\right\rangle \varphi_{j}(t) e_{j} \|_{H}=1 .
$$

Hence we have $\mathbf{T}=\left(e_{j} \otimes e_{j}\right)_{j} \in \widetilde{H}^{2}(\mathbb{T}, \mathcal{B}(H))$. On the other hand, since $\left\|T_{j}\right\|=1$ for all $j$, we have $\lim _{j \rightarrow \infty}\left\|T_{j}\right\|=1 \neq 0$, which implies that $\mathbf{T} \notin L^{1}(\mathbb{T}, \mathcal{B}(H))$ and so $\mathbf{T} \notin H_{0}^{2}(\mathbb{T}, \mathcal{B}(H))$, as desired.

Proposition 2.5. $\quad$ (i) $\widetilde{H}^{2}(\mathbb{T}, \mathcal{B}(H)) \subsetneq \ell_{\mathrm{SOT}}^{2}(\mathbb{N}, \mathcal{B}(H))$ and $\widetilde{H}^{2}\left(\mathbb{T}^{2}, \mathcal{B}(H)\right) \subsetneq \ell_{\mathrm{SOT}}^{2}\left(\mathbb{N}^{2}, \mathcal{B}(H)\right)$.

(ii) $\widetilde{H}^{2}(\mathbb{T}, \mathcal{B}(H)) \nsubseteq \ell^{2}(\mathbb{N}, \mathcal{B}(H))$ and $\ell^{2}(\mathbb{N}, \mathcal{B}(H)) \nsubseteq \widetilde{H}^{2}(\mathbb{T}, \mathcal{B}(H))$.

Proof. (i) Both inclusions are immediate from Plancherel's theorem (which holds for Hilbert-valued functions). It suffices to see that there exists $\mathbf{T} \in \ell_{\mathrm{SOT}}^{2}(\mathbb{N}, \mathcal{B}(H)) \backslash \widetilde{H}^{2}(\mathbb{T}, \mathcal{B}(H))$ because choosing matrices with a single row we obtain also a counterexample for the other inclusion. Now selecting $T_{n}=\widetilde{e_{n} \otimes x} \in \mathcal{B}(H)$ for a given $x \in H$ we clearly have $\mathbf{T}=\left(\widetilde{e_{n} \otimes x}\right)_{n} \in \ell_{\text {SOT }}^{2}(\mathbb{N}, \mathcal{B}(H))$ with $\|\mathbf{T}\|_{\ell_{\mathrm{SOT}}^{2}}(\mathbb{N}, \mathcal{B}(H))=\|x\|$. However, for any $t \in[0,2 \pi)$ and $N \in \mathbb{N}$,

$$
\left\|\sum_{n=1}^{N}\left(\widetilde{e_{n} \otimes x}\right) \varphi_{n}(t)\right\|_{B(H)}=\left\|\left(\sum_{n=1}^{N} e_{n} \varphi_{n}(t)\right) \otimes x\right\|_{\mathcal{B}(H)}=\|x\| \sqrt{N}
$$


showing that $\mathbf{T} \notin \widetilde{H}^{2}(\mathbb{T}, \mathcal{B}(H))$.

(ii) The example in Remark 2.4 shows that $\widetilde{H}^{2}(\mathbb{T}, \mathcal{B}(H)) \nsubseteq \ell^{2}(\mathbb{N}, \mathcal{B}(H))$. Let us now find $\mathbf{T} \in \ell^{2}(\mathbb{N}, \mathcal{B}(H)) \backslash \widetilde{H}^{2}(\mathbb{T}, \mathcal{B}(H))$. Consider $H=L^{2}(\mathbb{T})$ and $\mathbf{T}=\left(T_{j}\right)$ where $T_{j}: L^{2}(\mathbb{T}) \rightarrow L^{2}(\mathbb{T})$ is given by $T_{j}(f)=\frac{\varphi_{j}}{j} f$.

Clearly $\mathbf{T} \in \ell^{2}(\mathbb{N}, \mathcal{B}(H))$ since $\left\|T_{j}\right\|=1 / j$ for all $j \in \mathbb{N}$. On the other hand, for each $t \in[0,2 \pi)$ and $N \in \mathbb{N}$ one has that $\left(\sum_{j=1}^{N} T_{j} \varphi_{j}(t)\right)(f)=\left(\sum_{j=1}^{N} \frac{\varphi_{j}(t)}{j} \varphi_{j}\right) f$ and therefore

$$
\left\|\sum_{j=1}^{N} T_{j} \varphi_{j}(t)\right\|_{B(H)}=\left\|\sum_{j=1}^{N} \frac{\varphi_{j}(t)}{j} \varphi_{j}\right\|_{C(\mathbb{T})}=\sum_{j=1}^{N} \frac{1}{j} .
$$

This shows that $\mathbf{T} \notin \widetilde{H}^{2}(\mathbb{T}, \mathcal{B}(H))$.

\section{Preliminaries on regular vector measures}

We recall some facts for vector measures that can be found in [6, 7]. Let us consider the measure space $(\mathbb{T}, \mathfrak{B}(\mathbb{T}), m)$ where $\mathfrak{B}(\mathbb{T})$ stands for the Borel sets over $\mathbb{T}$ and $m$ for the Lebesgue measure on $\mathbb{T}$. Given a vector measure $\mu: \mathfrak{B}(\mathbb{T}) \rightarrow E$ and $B \in \mathfrak{B}(\mathbb{T})$, we shall denote $|\mu|(B)$ and $\|\mu\|(B)$ the variation and semi-variation of $\mu$ of the set $B$ given by

$$
|\mu|(B)=\sup \left\{\sum_{A \in \pi}\|\mu(A)\|, A \in \mathfrak{B}(\mathbb{T}), \pi \text { is a finite partition of } B\right\}
$$

and

$$
\|\mu\|(B)=\sup \left\{\left|\left\langle e^{*}, \mu\right\rangle\right|(B): e^{*} \in E^{*},\left\|e^{*}\right\|=1\right\},
$$

where $\left\langle e^{*}, \mu\right\rangle(A)=e^{*}(\mu(A))$ for all $A \in \mathfrak{B}(\mathbb{T})$. Of course $|\mu|(\cdot)$ becomes a positive measure on $\mathfrak{B}(\mathbb{T})$, while $\|\mu\|(\cdot)$ is only sub-additive in general. We shall denote $|\mu|=|\mu|(\mathbb{T})$ and $\|\mu\|=\|\mu\|(\mathbb{T})$. For dual spaces $E=F^{*}$ it is easy to see that $\|\mu\|=\sup \{|\langle\mu, f\rangle|: f \in$ $F,\|f\|=1\}$ where $\langle\mu, f\rangle(A)=\mu(A)(f)$.

In what follows we shall consider regular vector measures, that is to say vector measures $\mu: \mathfrak{B}(\mathbb{T}) \rightarrow E$ such that for each $\varepsilon>0$ and $B \in \mathfrak{B}(\mathbb{T})$ there exists a compact set $K$, an open set $O$ such that $K \subset B \subset O$ with $\|\mu\|(O \backslash K)<\varepsilon$. Let us denote by $\mathfrak{M}(\mathbb{T}, E)$ and $M(\mathbb{T}, E)$ the spaces of regular Borel measures with values in $E$ endowed with the norm given the semi-variation and variation respectively. Of course $M(\mathbb{T}, E) \subsetneq \mathfrak{M}(\mathbb{T}, E)$ when $E$ is infinite dimensional.

It is well known that the space $\mathfrak{M}(\mathbb{T}, E)$ can be identified with the space of weakly compact linear operators $T_{\mu}: C(\mathbb{T}) \rightarrow E$ and that $\left\|T_{\mu}\right\|=\|\mu\|$ (see $[6$, Chapter 6$]$ ). Hence, for each $\mu \in \mathfrak{M}(\mathbb{T}, E)$ and $k \in \mathbb{Z}$ we can define (see [3]) the $k$-Fourier coefficient by

$$
\widehat{\mu}(k)=T_{\mu}\left(\varphi_{-k}\right) .
$$


Also, the description of measures in $M(\mathbb{T}, E)$ can be done using absolutely summing operators (see [5]) and the variation can be described as the norm in such space (see [6]) but we shall not follow this approach. On the other hand, since we deal with either $E=\mathcal{B}(H)$ or $E=H$ we have at our disposal Singer's theorem (see for instance [8, 13, 14]), which in the case of dual spaces $E=F^{*}$ asserts that $M(\mathbb{T}, E)=C(\mathbb{T}, F)^{*}$. In other words there exists a bounded linear map $\Psi_{\mu}: C(\mathbb{T}, F) \rightarrow \mathbb{C}$ with $\left\|\Psi_{\mu}\right\|=|\mu|$ such that

$$
\Psi_{\mu}(y \phi)=T_{\mu}(\phi)(y), \quad \phi \in C(\mathbb{T}), y \in F
$$

In particular, for $k \in \mathbb{Z}$ one has $\widehat{\mu}(-k)(y)=\Psi_{\mu}\left(y \varphi_{k}\right)$ for each $y \in F$.

As mentioned above since $M\left(\mathbb{T}, \mathcal{L}\left(X, Y^{*}\right)\right)=C(\mathbb{T}, X \widehat{\otimes} Y)^{*}$, for each $\mu \in M\left(\mathbb{T}, \mathcal{L}\left(X, Y^{*}\right)\right)$ we can associate two operators $T_{\mu}$ and $\Psi_{\mu}$. Of course the connection between them is given by the formula

$$
T_{\mu}(\phi)(x)(y)=\Psi_{\mu}((x \otimes y) \phi), \quad \phi \in C(\mathbb{T}), x \in X, y \in Y .
$$

There is still one more possibility to be considered using the strong operator topology, namely $\Phi_{\mu}: C(\mathbb{T}, X) \rightarrow Y^{*}$ defined by

$$
\Phi_{\mu}(f)(y)=\Psi_{\mu}(f \otimes y), \quad f \in C(\mathbb{T}, X), y \in Y,
$$

where $f \otimes y(t)=f(t) \otimes y$.

Therefore, given $\mu \in \mathfrak{M}\left(\mathbb{T}, \mathcal{L}\left(X, Y^{*}\right)\right)$, we have three different linear operators defined on the corresponding spaces of polynomials: $T_{\mu}: \mathcal{P}(\mathbb{T}) \rightarrow \mathcal{L}\left(X, Y^{*}\right), \Psi_{\mu}: \mathcal{P}(\mathbb{T}, X \widehat{\otimes} Y) \rightarrow \mathbb{C}$ and $\Phi_{\mu}: \mathcal{P}(\mathbb{T}, X) \rightarrow Y^{*}$ defined by the formulae

$$
\begin{gathered}
T_{\mu}\left(\sum_{j=-M}^{N} \alpha_{j} \varphi_{j}\right)=\sum_{j=-M}^{N} \alpha_{j} \widehat{\mu}(-j), \quad N, M \in \mathbb{N}, \alpha_{j} \in \mathbb{C}, \\
\Psi_{\mu}\left(\sum_{j=-M}^{N}\left(\sum_{n=1}^{n_{j}} x_{j n}\right) \otimes\left(\sum_{m=1}^{m_{j}} y_{j m}\right) \varphi_{j}\right)=\sum_{j=-M}^{N}\left(\sum_{n=1}^{n_{j}} \sum_{m=1}^{m_{j}} \widehat{\mu}(-j)\left(x_{j n}\right)\left(y_{j m}\right)\right), \\
\Phi_{\mu}\left(\sum_{j=-M}^{N} x_{j} \varphi_{j}\right)=\sum_{j=-M}^{N} \widehat{\mu}(-j)\left(x_{j}\right), \quad N, M \in \mathbb{N}, x_{j} \in X .
\end{gathered}
$$

When restricting to the case $Y^{*}=H$ we obtain the following connection between them:

$$
\mathcal{J} T_{\mu}(\psi)(x \otimes y)=\Psi_{\mu}((x \otimes y) \psi)=\left\langle\Phi_{\mu}(x \psi), y\right\rangle, \quad \psi \in \mathcal{P}(\mathbb{T}), x, y \in H
$$

Given $\mu \in \mathfrak{M}\left(\mathbb{T}, \mathcal{L}\left(X, Y^{*}\right)\right)$ and $x \in X$, let us denote by $\mu_{x}$ the $Y^{*}$-valued measure given by

$$
\mu_{x}(A)=\mu(A)(x), \quad A \in \mathfrak{B}(\mathbb{T})
$$


It is elementary to see that $\mu_{x}$ is a regular measure because one can associate the weakly compact operator $T_{\mu_{x}}=\delta_{x} \circ T_{\mu}: C(\mathbb{T}) \rightarrow Y^{*}$ where $\delta_{x}$ stands for the operator $\delta_{x}: \mathcal{L}\left(X, Y^{*}\right)$ $\rightarrow Y^{*}$ given by $\delta_{x}(T)=T(x)$ for $T \in \mathcal{L}\left(X, Y^{*}\right)$.

If $\mu \in \mathfrak{M}(\mathbb{T}, \mathcal{B}(H)), k \in \mathbb{Z}$ and $x, y \in H$ then $\mu_{x} \in \mathfrak{M}(\mathbb{T}, H)$,

$$
\left\langle\mu_{x}(A), y\right\rangle=\mathcal{J} \mu(A)(x \otimes y), \quad A \in \mathfrak{B}(\mathbb{T})
$$

and

$$
\langle\widehat{\mu}(k)(x), y\rangle=\left\langle\widehat{\mu}_{x}(k), y\right\rangle=\mathcal{J} \widehat{\mu}(k)(x \otimes y) .
$$

Let us introduce a new space of measures appearing in the case $E=\mathcal{B}(H)$.

Definition 3.1. Let $\mu \in \mathfrak{M}(\mathbb{T}, \mathcal{B}(H))$. We say that $\mu \in M_{\mathrm{SOT}}(\mathbb{T}, \mathcal{B}(H))$ if $\mu_{x} \in M(\mathbb{T}, H)$ for any $x \in H$. We write

$$
\|\mu\|_{\mathrm{SOT}}=\sup \left\{\left|\mu_{x}\right|: x \in H,\|x\|=1\right\} .
$$

Proposition 3.2. $M(\mathbb{T}, \mathcal{B}(H)) \subsetneq M_{\mathrm{SOT}}(\mathbb{T}, \mathcal{B}(H)) \subsetneq \mathfrak{M}(\mathbb{T}, \mathcal{B}(H))$.

Proof. The inclusions between the spaces follow from the inequalities

$$
|\langle\mu(A)(x), y\rangle| \leq\|\mu(A)(x)\|\|y\| \leq\|\mu(A)\|\|x\|\|y\|
$$

which leads to

$$
\left|\left\langle\mu_{x}, y\right\rangle\right| \leq\left|\mu_{x}\right|\|y\| \leq|\mu|\|x\|\|y\|
$$

and the corresponding embeddings with norm 1 trivially follow.

Let $H=\ell^{2}$. We shall find measures $\mu_{1} \in M_{\mathrm{SOT}}(\mathbb{T}, \mathcal{B}(H)) \backslash M(\mathbb{T}, \mathcal{B}(H))$ and $\mu_{2} \in$ $\mathfrak{M}(\mathbb{T}, \mathcal{B}(H)) \backslash M_{\mathrm{SOT}}(\mathbb{T}, \mathcal{B}(H))$. Both can be constructed relying on a similar argument. Let $y_{0} \in H$ with $\left\|y_{0}\right\|=1$ and select a Hilbert-valued regular measure $\nu$ with $|\nu|=\infty$ (for instance take a Pettis integrable, but not Bochner integrable function $f: \mathbb{T} \rightarrow H$ given by $t \rightarrow\left(f_{n}(t)\right)_{n}$ and $\nu(A)=\left(\int_{A} f_{n}(t) \frac{d t}{2 \pi}\right)_{n}$ for $\left.A \in \mathfrak{B}(\mathbb{T})\right)$. Denote $T_{\nu}: C(\mathbb{T}) \rightarrow H$ the corresponding bounded (and hence weakly compact) operator associated to $\nu$ with $\left\|T_{\nu}\right\|=\|\nu\|$.

Define

$$
\mu_{1}(A)(x)=\langle x, \nu(A)\rangle y_{0}, \quad A \in \mathfrak{B}(\mathbb{T})
$$

and

$$
\mu_{2}(A)(x)=\left\langle x, y_{0}\right\rangle \nu(A), \quad A \in \mathfrak{B}(\mathbb{T})
$$

In other words, if $J_{y}: H \rightarrow \mathcal{B}(H)$ and $I_{y}: H \rightarrow \mathcal{B}(H)$ stand for the operators

$$
J_{y}(x)(z)=\langle z, x\rangle y, \quad I_{y}(x)(z)=\langle x, y\rangle z, \quad x, y, z \in H,
$$


then we have that $T_{\mu_{1}}=J_{y_{0}} T_{\nu}$ and $T_{\mu_{2}}=I_{y_{0}} T_{\nu}$ are weakly compact. Hence $\mu_{1}, \mu_{2} \in$ $\mathfrak{M}(\mathbb{T}, \mathcal{B}(H))$.

Note that $\left|\left(\mu_{1}\right)_{x}\right|=|\langle x, \nu\rangle|$ and $\left|\left(\mu_{2}\right)_{x}\right|=\left|\left\langle x, y_{0}\right\rangle\right||\nu|, x \in H$. Hence

$$
\left\|\mu_{1}\right\|_{\mathrm{SOT}}=\|\nu\|, \quad\left\|\mu_{2}\right\|_{\mathrm{SOT}}=|\nu| .
$$

Also notice that $\left\|\mu_{1}(A)\right\|_{\mathcal{B}(H)}=\|\nu(A)\|_{H}$, and therefore $\left|\mu_{1}\right|=|\nu|$, which gives the desired results.

Definition 3.3. Let $\mu: \mathfrak{B}(\mathbb{T}) \rightarrow \mathcal{L}\left(X, Y^{*}\right)$ be a vector measure. We define "the adjoint measure" $\mu^{*}: \mathfrak{B}(\mathbb{T}) \rightarrow \mathcal{L}\left(Y, X^{*}\right)$ by the formula

$$
\mu^{*}(A)(y)(x)=\mu_{x}(A)(y), \quad A \in \mathfrak{B}(\mathbb{T}), x \in X, y \in Y
$$

In the case that $\mu \in \mathfrak{M}(\mathbb{T}, \mathcal{B}(H))$ with the identification $Y^{*}=H$, one clearly has that

$$
\left\langle x, \mu^{*}(A)(y)\right\rangle=\langle\mu(A)(x), y\rangle, \quad A \in \mathfrak{B}(\mathbb{T}), x, y \in H
$$

Remark 3.4. $\mu^{*}$ belongs to $\mathfrak{M}(\mathbb{T}, \mathcal{B}(H))$ (resp. $M(\mathbb{T}, \mathcal{B}(H))$ ) if and only if $\mu$ belongs to $\mathfrak{M}(\mathbb{T}, \mathcal{B}(H))(\operatorname{resp} . M(\mathbb{T}, \mathcal{B}(H)))$. Moreover $\|\mu\|=\left\|\mu^{*}\right\|\left(\right.$ resp. $\left.|\mu|=\left|\mu^{*}\right|\right)$.

The results follow using that $T_{\mu^{*}}(\phi)=\left(T_{\mu}(\phi)\right)^{*}$ for any $\phi \in C(\mathbb{T})$ and $\|\mu(A)\|=$ $\left\|\mu^{*}(A)\right\|$ for any $A \in \mathfrak{B}(\mathbb{T})$.

Let us describe the norm in $M_{\mathrm{SOT}}(\mathbb{T}, \mathcal{B}(H)$ using the adjoint measure.

Proposition 3.5. Let $\mu \in \mathfrak{M}(\mathbb{T}, \mathcal{B}(H))$. Then $\mu \in M_{\mathrm{SOT}}(\mathbb{T}, \mathcal{B}(H))$ if and only if $\Phi_{\mu^{*}} \in$ $\mathcal{L}(C(\mathbb{T}, H), H)$. Moreover $\|\mu\|_{\mathrm{SOT}}=\left\|\Phi_{\mu^{*}}\right\|$.

Proof. By definition, $\mu \in M_{\mathrm{SOT}}(\mathbb{T}, \mathcal{B}(H))$ if and only if the operator $S_{\mu}(x)=\mu_{x}$ is well defined and belongs to $\mathcal{L}(H, M(\mathbb{T}, H))$. Moreover, $\|\mu\|_{\mathrm{SOT}}=\left\|S_{\mu}\right\|$. The result follows if we show that $S_{\mu}$ is the adjoint of $\Phi_{\mu^{*}}$. Recall that, identifying $H=H^{*}$, we have $\mu^{*} \in \mathfrak{M}(\mathbb{T}, \mathcal{B}(H))$. Hence $\Phi_{\mu^{*}}: \mathcal{P}(\mathbb{T}, H) \rightarrow H$ is generated by linearity using

$$
\Phi_{\mu^{*}}\left(x \varphi_{k}\right)=\widehat{\mu^{*}}(-k)(x)=\widehat{\mu}(-k)^{*}(x), \quad x \in H, k \in \mathbb{Z} .
$$

Therefore, if $k \in \mathbb{Z}, x, y \in H$, since $M(\mathbb{T}, H)=(C(\mathbb{T}, H))^{*}$, we have

$$
S_{\mu}(y)\left(x \varphi_{k}\right)=\Psi_{\mu_{y}}\left(x \varphi_{k}\right)=\left\langle\widehat{\mu_{y}}(-k), x\right\rangle=\langle\widehat{\mu}(-k)(y), x\rangle=\left\langle y, \Phi_{\mu^{*}}\left(x \varphi_{k}\right)\right\rangle .
$$

By linearity we extend to $\left\langle y, \Phi_{\mu^{*}}(x \phi)\right\rangle=S_{\mu}(y)(x \phi)$ for any polynomial $\phi$ and since $\mathcal{P}(\mathbb{T}, H)$ is dense in $C(\mathbb{T}, H)$ we obtain the result. This completes the proof.

Let us consider the following subspace of regular measures which plays an important role in what follows. 
Definition 3.6. Let us write $V^{\infty}(\mathbb{T}, E)$ for the subspace of those measures $\mu \in \mathfrak{M}(\mathbb{T}, E)$ such that there exists $C>0$ with

$$
\|\mu(A)\| \leq C m(A), \quad A \in \mathfrak{B}(\mathbb{T}) .
$$

We define

$$
\|\mu\|_{\infty}=\sup \left\{\frac{\|\mu(A)\|}{m(A)}: m(A)>0\right\}
$$

It is clear that any $\mu \in V^{\infty}(\mathbb{T}, \mathcal{B}(H))$ also belongs to $M(\mathbb{T}, \mathcal{B}(H))$ and it is absolutely continuous with respect to $m$.

Let us point out two more possible descriptions of $V^{\infty}(\mathbb{T}, E)$. One option is to look at $V^{\infty}(\mathbb{T}, E)=\mathcal{L}\left(L^{1}(\mathbb{T}), E\right)$ (see $[7$, page 261$]$ ), that is to say that $T_{\mu}$ has a bounded extension to $L^{1}(\mathbb{T})$. Hence a measure $\mu \in \mathfrak{M}(\mathbb{T}, E)$ belongs to $V^{\infty}(\mathbb{T}, E)$ if and only if

$$
\left\|T_{\mu}(\psi)\right\| \leq C\|\psi\|_{L^{1}(\mathbb{T})}, \quad \psi \in C(\mathbb{T})
$$

Moreover $\left\|T_{\mu}\right\|_{L^{1}(\mathbb{T}) \rightarrow E}=\|\mu\|_{\infty}$.

In the case that $E=F^{*}$ also one has that $V^{\infty}(\mathbb{T}, E)=L^{1}(\mathbb{T}, F)^{*}$, that is the dual of the space of Bochner integrable functions. In this case a measure $\mu \in V^{\infty}(\mathbb{T}, E)$ if and only if $\Psi_{\mu}$ has a bounded extension to $L^{1}(\mathbb{T}, F)^{*}$, that is

$$
\left\|\Psi_{\mu}(p)\right\| \leq C\|p\|_{L^{1}(\mathbb{T}, F)}, \quad p \in \mathcal{P}(\mathbb{T}, F) .
$$

Moreover $\left\|\Psi_{\mu}\right\|_{L^{1}(\mathbb{T}, F)^{*}}=\|\mu\|_{\infty}$.

Although measures in $V^{\infty}(\mathbb{T}, \mathcal{B}(H))$ are absolutely continuous with respect to $m$, the reader should be aware that they might not have a Radon-Nikodym derivative in $L^{1}(\mathbb{T}, E)$ (see 6, Chapter 3]).

For the sake of completeness we give an example for $E=\mathcal{B}(H)$ of such a situation.

Proposition 3.7. Let $H=\ell^{2}$ and $\mu \in \mathfrak{M}(\mathbb{T}, \mathcal{B}(H))$ such that $T_{\mu} \in \mathcal{L}(C(\mathbb{T}), \mathcal{B}(H))$ is given by

$$
T_{\mu}(\phi)=\sum_{n=1}^{\infty} \widehat{\phi}(n) \widetilde{e_{n} \otimes e_{n}} .
$$

Then $\mu \in V^{\infty}(\mathbb{T}, \mathcal{B}(H))$ with $\|\mu\|_{\infty}=1$,

$$
\widehat{\mu}(k)= \begin{cases}\widehat{e_{k} \otimes e_{k}} & \text { if } k \geq 1, \\ 0 & \text { if } k \leq 0\end{cases}
$$

but it does not have a Radon-Nikodym derivative in $L^{1}(\mathbb{T}, \mathcal{B}(H))$. 
Proof. Let us show that $T_{\mu}$ defines a continuous operator from $L^{1}(\mathbb{T})$ to $\mathcal{B}(H)$ with norm 1 . In such a case, using that the inclusion $C(\mathbb{T}) \rightarrow L^{1}(\mathbb{T})$ is weakly compact, one automatically has that $\mu \in \mathfrak{M}(\mathbb{T}, \mathcal{B}(H))$. For $x=\left(\alpha_{n}\right) \in H$ and $y=\left(\beta_{n}\right) \in H$ one has

$$
\left|\left\langle T_{\mu}(\phi)(x), y\right\rangle\right|=\left|\sum_{n=1}^{\infty} \widehat{\phi}(n) \alpha_{n} \beta_{n}\right| \leq \sup _{n \geq 1}|\widehat{\phi}(n)|\|x\|\|y\| \leq\|\phi\|_{L^{1}(\mathbb{T})}\|x\|\|y\| .
$$

This gives that $\mu \in V^{\infty}(\mathbb{T}, \mathcal{B}(H))$ and $\|\mu\|_{\infty} \leq 1$. Using that $T_{\mu}\left(\varphi_{j}\right)=\widetilde{e_{j} \otimes e_{j}}$ and $\left\|\widetilde{e_{j} \otimes e_{j}}\right\|_{\mathcal{B}(H)}=1$ we get the equality of norms.

The result on Fourier coefficients is obvious. To show that $\mu$ does not have a Bochner integrable Radon-Nikodym derivative follows now using that otherwise $\widehat{\mu}(k)=\widehat{f}(k)$ for some $f \in L^{1}(\mathbb{T}, \mathcal{B}(H))$ which implies that $\|\widehat{f}(k)\| \rightarrow 0$ as $k \rightarrow \infty$ while $\|\widehat{\mu}(k)\|=1$ for $k \geq 1$. This completes the proof.

We finish this section with a known characterization of measures in $M\left(\mathbb{T}, F^{*}\right)$ to be used later on, that we include for sake of completeness.

Lemma 3.8. Let $E=F^{*}$ be a dual Banach space and $\mu \in \mathfrak{M}(\mathbb{T}, E)$. For each $0<r<1$ we define

$$
P_{r} * \mu(t)=\sum_{k \in \mathbb{Z}} \widehat{\mu}(k) r^{|k|} \varphi_{k}(t), \quad t \in[0,2 \pi) .
$$

Then

(i) $P_{r} * \mu \in C(\mathbb{T}, E)$ and $\left\|P_{r} * \mu\right\|_{C(\mathbb{T}, E)} \leq\|\mu\| \frac{1+r}{1-r}$ for any $0<r<1$.

(ii) $\mu \in M(\mathbb{T}, E)$ if and only if $\sup _{0<r<1}\left\|P_{r} * \mu\right\|_{L^{1}(\mathbb{T}, E)}<\infty$. Moreover

$$
|\mu|=\sup _{0<r<1}\left\|P_{r} * \mu\right\|_{L^{1}(\mathbb{T}, E)} .
$$

Proof. (i) Observe that

$$
\sum_{k \in \mathbb{Z}}|\widehat{\mu}(k)| r^{|k|}\left\|\varphi_{k}\right\|_{C(\mathbb{T})} \leq\left\|T_{\mu}\right\|\left(1+2 \sum_{k=1}^{\infty} r^{k}\right)=\|\mu\| \frac{1+r}{1-r} .
$$

This shows that the series in (3.1) is absolutely convergent in $C(\mathbb{T}, E)$ and we obtain (i).

(ii) Assume that $\mu \in M(\mathbb{T}, E)$. In particular $|\mu| \in M(\mathbb{T})$ and

$$
\int_{0}^{2 \pi}\left\|P_{r} * \mu(t)\right\| \frac{d t}{2 \pi} \leq \int_{0}^{2 \pi} P_{r} *|\mu|(t) \frac{d t}{2 \pi} .
$$

Hence, using the scalar-valued result, we have

$$
\sup _{0<r<1}\left\|P_{r} * \mu\right\|_{L^{1}(\mathbb{T}, E)} \leq \sup _{0<r<1}\left\|P_{r} *|\mu|\right\|_{L^{1}(\mathbb{T})} \leq \sup _{0<r<1}|\mu|\left\|P_{r}\right\|_{L^{1}(\mathbb{T})}=|\mu|
$$


Conversely, assume that $\sup _{0<r<1}\left\|P_{r} * \mu\right\|_{L^{1}(\mathbb{T}, E)}<\infty$. Since $L^{1}(\mathbb{T}, E) \subseteq M(\mathbb{T}, E)=$ $C(\mathbb{T}, F)^{*}$, from the Banach-Alaoglu theorem one can find a sequence $r_{n}$ converging to 1 and a measure $\nu \in M(\mathbb{T}, E)$ such that $P_{r_{n}} * \mu \rightarrow \nu$ in the $w^{*}$-topology. Selecting now functions in $C(\mathbb{T}, F)$ given by $y \varphi_{-k}$ for all $y \in F$ and $k \in \mathbb{Z}$ one shows that $\widehat{\nu}(k)=\widehat{\mu}(k)$. This gives that $\mu=\nu$ and therefore $\mu \in M(\mathbb{T}, E)$. Finally, notice that

$$
|\mu|=\sup \left\{\left|\Psi_{\mu}(p)\right|: p \in \mathcal{P}(\mathbb{T}, F),\|p\|_{C(\mathbb{T}, F)}=1\right\} .
$$

Given now $p=\sum_{k=-M}^{N} y_{k} \varphi_{k}$, one has $P_{r} * p=\sum_{k=-M}^{N} y_{k} r^{|k|} \varphi_{k}$ and

$$
\Psi_{\mu}\left(P_{r} * p\right)=\sum_{k=-M}^{N} \widehat{\mu}(k)\left(y_{k}\right) r^{|k|}=\int_{0}^{2 \pi} P_{r} * \mu(t)(p(t)) \frac{d t}{2 \pi} .
$$

Finally, since $p=\lim _{r \rightarrow 1} P_{r} * p$ is in $C(\mathbb{T}, F)$, we have

$$
\begin{aligned}
\left|\Psi_{\mu}(p)\right| & =\lim _{r \rightarrow 1}\left|\Psi_{\mu}\left(P_{r} * p\right)\right| \\
& \leq \sup _{0<r<1}\left|\int_{0}^{2 \pi} P_{r} * \mu(t)(p(t)) \frac{d t}{2 \pi}\right| \\
& \leq \sup _{0<r<1}\left\|P_{r} * \mu\right\|_{L^{1}(\mathbb{T}, E)}\|p\|_{C(\mathbb{T}, F)} .
\end{aligned}
$$

This gives the inequality $|\mu| \leq \sup _{0<r<1}\left\|P_{r} * \mu\right\|_{L^{1}(\mathbb{T}, E)}$ and the proof is complete.

\section{Some results on matrices of operators}

Throughout the rest of the paper, we write $\mathbf{A}=\left(T_{k j}\right) \subset \mathcal{B}(H), \mathbf{R}_{k}$ and $\mathbf{C}_{j}$ the $k$-row respectively, that is

$$
\mathbf{R}_{k}=\left(T_{k j}\right)_{j=1}^{\infty}, \quad \mathbf{C}_{j}=\left(T_{k j}\right)_{k=1}^{\infty}
$$

and

$$
\mathbf{A}_{N, M}(s, t)=\sum_{k=1}^{M} \sum_{j=1}^{N} T_{k j} \overline{\varphi_{j}(s)} \varphi_{k}(t), \quad 0 \leq t, s<2 \pi, N, M \in \mathbb{N} .
$$

For each $\mathbf{x}=\left(x_{j}\right) \in \ell^{2}(H)$ we consider the function $h_{\mathbf{x}}$ given by

$$
h_{\mathbf{x}}(t)=\sum_{j=1}^{\infty} x_{j} \varphi_{j}(t), \quad t \in[0,2 \pi) .
$$

Remark 4.1. Observe that $\mathbf{A} \in \widetilde{H}^{2}\left(\mathbb{T}^{2}, \mathcal{B}(H)\right)$ if and only if

$$
\sup _{N, M}\left\|\mathbf{A}_{N, M}\right\|_{L^{2}\left(\mathbb{T}^{2}, \mathcal{B}(H)\right)}<\infty .
$$

Note that $\mathbf{x} \in \ell^{2}(H)$ if and only if $h_{\mathbf{x}} \in H_{0}^{2}(\mathbb{T}, H)$. Moreover

$$
\|\mathbf{x}\|_{\ell^{2}(H)}=\left\|h_{\mathbf{x}}\right\|_{H^{2}(\mathbb{T}, H)} .
$$


Proposition 4.2. Let $\mathbf{A}=\left(T_{k j}\right) \subset \mathcal{B}(H)$.

(i) If $\mathbf{A} \in \ell_{\mathrm{SOT}}^{2}\left(\mathbb{N}^{2}, \mathcal{B}(H)\right)$ then $\mathbf{R}_{k}, \mathbf{C}_{j} \in \ell_{\mathrm{SOT}}^{2}(\mathbb{N}, \mathcal{B}(H))$ for all $k, j \in \mathbb{N}$.

(ii) If $\mathbf{A} \in \widetilde{H}^{2}\left(\mathbb{T}^{2}, \mathcal{B}(H)\right)$ then $\mathbf{C}_{j}, \mathbf{R}_{k} \in \widetilde{H}^{2}(\mathbb{T}, \mathcal{B}(H))$ for all $j, k \in \mathbb{N}$.

Proof. (i) follows trivially from the definitions.

(ii) Let $k^{\prime} \in \mathbb{N}, M \in \mathbb{N}$ and $t \in[0,2 \pi)$. For $N \geq k^{\prime}$ we have

$$
\sum_{j=1}^{N} T_{k^{\prime} j} \varphi_{j}(t)=\int_{0}^{2 \pi}\left(\sum_{k=1}^{N} \sum_{j=1}^{M} T_{k j} \varphi_{j}(t) \varphi_{k}(s)\right) \overline{\varphi_{k^{\prime}}(s)} \frac{d s}{2 \pi} .
$$

Therefore

$$
\int_{0}^{2 \pi}\left\|\sum_{j=1}^{N} T_{k^{\prime} j} \varphi_{j}(t)\right\|^{2} \frac{d t}{2 \pi} \leq \int_{0}^{2 \pi} \int_{0}^{2 \pi}\left\|\sum_{k=1}^{N} \sum_{j=1}^{M} T_{k j} \varphi_{j}(t) \varphi_{k}(s)\right\|^{2} \frac{d s}{2 \pi} \frac{d t}{2 \pi} .
$$

Hence $\left\|\mathbf{R}_{k^{\prime}}\right\|_{\widetilde{H}^{2}(\mathbb{T}, \mathcal{B}(H))} \leq\|\mathbf{A}\|_{\widetilde{H}^{2}\left(\mathbb{T}^{2}, \mathcal{B}(H)\right)}$. A similar argument shows that $\left\|\mathbf{C}_{j}\right\|_{\widetilde{H}^{2}(\mathbb{T}, \mathcal{B}(H))}$ $\leq\|\mathbf{A}\|_{\widetilde{H}^{2}\left(\mathbb{T}^{2}, \mathcal{B}(H)\right)}$ and it is left to the reader.

Definition 4.3. Let $\mathbf{A}=\left(T_{k j}\right) \subset \mathcal{B}(H)$. Define $B_{\mathbf{A}}: \mathcal{P}_{a}(\mathbb{T}, H) \times \mathcal{P}_{a}(\mathbb{T}, H) \rightarrow \mathbb{C}$ by

$$
\left(h_{\mathbf{x}}, h_{\mathbf{y}}\right) \rightarrow \int_{0}^{2 \pi} \int_{0}^{2 \pi} \mathcal{J} \mathbf{A}_{N, M}(s, t)\left(h_{\mathbf{x}}(s) \otimes h_{\mathbf{y}}(t)\right) \frac{d s}{2 \pi} \frac{d t}{2 \pi}
$$

where $h_{\mathbf{x}}=\sum_{j=1}^{N} x_{j} \varphi_{j}$ and $h_{\mathbf{y}}=\sum_{k=1}^{M} y_{k} \varphi_{k}$ for $x_{j}, y_{k} \in H$.

We now give the characterization of bounded operators in $\mathcal{B}\left(\ell^{2}(H)\right)$ in terms of bilinear maps.

Proposition 4.4. If $\mathbf{A}=\left(T_{k j}\right) \subset \mathcal{B}(H)$ then

$$
\langle\langle\mathbf{A}(\mathbf{x}), \mathbf{y}\rangle\rangle=B_{\mathbf{A}}\left(h_{\mathbf{x}}, h_{\mathbf{y}}\right), \quad \mathbf{x}, \mathbf{y} \in c_{00}(H) .
$$

In particular, $\mathbf{A} \in \mathcal{B}\left(\ell^{2}(H)\right)$ if and only if $B_{\mathbf{A}}$ extends to a bounded bilinear map on $H_{0}^{2}(\mathbb{T}, H) \times H_{0}^{2}(\mathbb{T}, H)$. Moreover $\|\mathbf{A}\|=\left\|B_{\mathbf{A}}\right\|$.

Proof. To show 4.1] we observe that for $h_{\mathbf{x}}=\sum_{j=1}^{N} x_{j} \varphi_{j}$ and $h_{\mathbf{y}}=\sum_{k=1}^{M} y_{k} \varphi_{k}$ we have 


$$
\begin{aligned}
y_{k}=\int_{0}^{2 \pi} h_{\mathbf{y}}(t) \overline{\varphi_{k}(t)} \frac{d t}{2 \pi} \text { and } x_{j} & =\int_{0}^{2 \pi} h_{\mathbf{x}}(t) \overline{\varphi_{j}(s)} \frac{d s}{2 \pi} \text {. Hence } \\
\sum_{k=1}^{M}\left\langle\sum_{j=1}^{N} T_{k j} x_{j}, y_{k}\right\rangle & =\int_{0}^{2 \pi}\left\langle\sum_{k=1}^{M}\left(\sum_{j=1}^{N} T_{k j} x_{j}\right) \varphi_{k}(t), h_{\mathbf{y}}(t)\right\rangle \frac{d t}{2 \pi} \\
& =\int_{0}^{2 \pi}\left\langle\sum_{k=1}^{M}\left(\sum_{j=1}^{N} T_{k j} \varphi_{k}(t)\right)\left(x_{j}\right), h_{\mathbf{y}}(t)\right\rangle \frac{d t}{2 \pi} \\
& =\int_{0}^{2 \pi}\left\langle\int_{0}^{2 \pi} \mathbf{A}_{N, M}(s, t)\left(h_{\mathbf{x}}(s)\right) \frac{d s}{2 \pi}, h_{\mathbf{y}}(t)\right\rangle \frac{d t}{2 \pi} \\
& =\int_{0}^{2 \pi} \int_{0}^{2 \pi} \mathcal{J} \mathbf{A}_{N, M}(s, t)\left(h_{\mathbf{x}}(s) \otimes h_{\mathbf{y}}(t)\right) \frac{d s}{2 \pi} \frac{d t}{2 \pi} .
\end{aligned}
$$

The equality of norms follows trivially.

From Proposition 4.4 one can produce some sufficient conditions for $\mathbf{A}$ to belong to $\mathcal{B}\left(\ell^{2}(H)\right)$.

Corollary 4.5. If $\mathbf{A} \in \widetilde{H}^{2}\left(\mathbb{T}^{2}, \mathcal{B}(H)\right) \cup \ell^{2}\left(\mathbb{N}^{2}, \mathcal{B}(H)\right)$ then $\mathbf{A} \in \mathcal{B}\left(\ell^{2}(H)\right)$ and $\|\mathbf{A}\| \leq$ $\min \left\{\|\mathbf{A}\|_{\widetilde{H}^{2}\left(\mathbb{T}^{2}, \mathcal{B}(H)\right)},\|\mathbf{A}\|_{\ell^{2}\left(\mathbb{N}^{2}, \mathcal{B}(H)\right)}\right\}$.

Proof. Assume first $\mathbf{A} \in \ell^{2}\left(\mathbb{N}^{2}, \mathcal{B}(H)\right)$. Then

$$
|\langle\langle\mathbf{A}(\mathbf{x}), \mathbf{y}\rangle\rangle| \leq \sum_{k=1}^{\infty} \sum_{j=1}^{\infty}\left\|T_{k j}\right\|\left\|x_{j}\right\|\left\|y_{k}\right\|
$$

and therefore, using Cauchy-Schwarz's inequality in $\ell^{2}\left(\mathbb{N}^{2}\right)$,

$$
\begin{aligned}
|\langle\mathbf{A}(\mathbf{x}), \mathbf{y}\rangle\rangle \mid & \leq\|\mathbf{A}\|_{\ell^{2}\left(\mathbb{N}^{2}, \mathcal{B}(H)\right)}\left\|\left(\left\|x_{j}\right\|\left\|y_{k}\right\|\right)\right\|_{\ell^{2}\left(\mathbb{N}^{2}\right)} \\
& =\|\mathbf{A}\|_{\ell^{2}\left(\mathbb{N}^{2}, \mathcal{B}(H)\right)}\|\mathbf{x}\|\|\mathbf{y}\| .
\end{aligned}
$$

Assume now $\mathbf{A} \in \widetilde{H}^{2}\left(\mathbb{T}^{2}, \mathcal{B}(H)\right)$ and apply Cauchy-Schwarz in $L^{2}\left(\mathbb{T}^{2}\right)$

$$
\begin{aligned}
& \left|\int_{0}^{2 \pi} \int_{0}^{2 \pi} \mathcal{J} \mathbf{A}_{N, M}(s, t)\left(h_{\mathbf{x}}(s) \otimes h_{\mathbf{y}}(t)\right) \frac{d s}{2 \pi} \frac{d t}{2 \pi}\right| \\
\leq & \left\|\mathbf{A}_{N, M}\right\|_{H_{0}^{2}\left(\mathbb{T}^{2}, \mathcal{B}(H)\right)}\left\|h_{\mathbf{x}}\right\|_{H_{0}^{2}(\mathbb{T}, H)}\left\|h_{\mathbf{y}}\right\|_{H_{0}^{2}(\mathbb{T}, H)} .
\end{aligned}
$$

Now the result follows from Proposition 4.4 .

Actually a sufficient condition better than $\mathbf{A} \in \ell^{2}\left(\mathbb{N}^{2}, \mathcal{B}(H)\right)$ is given in the following result.

Proposition 4.6. Let $\mathbf{A}=\left(T_{k j}\right) \subset \mathcal{B}(H)$ such that $\mathbf{C}_{j}$ for all $j \in \mathbb{N}$ or $\mathbf{R}_{k}^{*} \in \ell_{\mathrm{SOT}}^{2}(\mathbb{N}, \mathcal{B}(H))$ for all $k \in \mathbb{N}$ and satisfy

$$
\min \left\{\left\|\left(\mathbf{C}_{j}\right)\right\|_{\ell^{2}\left(\mathbb{N}, \ell_{\mathrm{SOT}}^{2}(\mathbb{N}, \mathcal{B}(H))\right)},\left\|\left(\mathbf{R}_{k}^{*}\right)\right\|_{\ell^{2}\left(\mathbb{N}, \ell_{\mathrm{SOT}}^{2}(\mathbb{N}, \mathcal{B}(H))\right)}\right\}=M<\infty .
$$

Then $\mathbf{A} \in \mathcal{B}\left(\ell^{2}(H)\right)$ and $\|\mathbf{A}\| \leq M$. 
Proof. Let $\mathbf{x}, \mathbf{y} \in \ell^{2}(H)$, we have

$$
\begin{aligned}
|\langle\mathbf{A}(\mathbf{x}), \mathbf{y}\rangle\rangle \mid & \leq \sum_{k=1}^{\infty} \sum_{j=1}^{\infty}\left\|y_{k}\right\|\left\|T_{k j}\left(\frac{x_{j}}{\left\|x_{j}\right\|}\right)\right\|\left\|x_{j}\right\| \\
& \leq\left(\sum_{j=1}^{\infty} \sum_{k=1}^{\infty}\left\|T_{k j}\left(\frac{x_{j}}{\left\|x_{j}\right\|}\right)\right\|^{2}\right)^{1 / 2}\left(\sum_{k=1}^{\infty} \sum_{j=1}^{\infty}\left\|y_{k}\right\|^{2}\left\|x_{j}\right\|^{2}\right)^{1 / 2} \\
& \leq\|\mathbf{x}\|_{\ell^{2}(H)}\|\mathbf{y}\|_{\ell^{2}(H)}\left(\sum_{j=1}^{\infty}\left\|\mathbf{C}_{j}\right\|_{\ell_{\text {SOT }}^{2}(\mathbb{N}, \mathcal{B}(H))}^{1 / 2}\right)^{1}
\end{aligned}
$$

Similar argument works with $\mathbf{R}_{k}^{*}$, which completes the proof.

Let us now present some necessary conditions for $\mathbf{A} \in \mathcal{B}\left(\ell^{2}(H)\right)$. Since $\left\langle\left\langle\mathbf{A}\left(x \mathbf{e}_{j}\right), y \mathbf{e}_{k}\right\rangle\right\rangle$ $=\left\langle T_{k j}(x), y\right\rangle$, we have that if $\mathbf{A} \in \mathcal{B}\left(\ell^{2}(H)\right)$ then $\mathbf{A} \in \ell^{\infty}\left(\mathbb{N}^{2}, \mathcal{B}(H)\right)$ and $\sup _{k, j}\left\|T_{k j}\right\| \leq$ $\|\mathbf{A}\|$.

Lemma 4.7. Let $\mathbf{A}=\left(T_{k j}\right) \in \mathcal{B}\left(\ell^{2}(H)\right)$. Then $\left(\mathbf{C}_{j}\right)_{j},\left(\mathbf{R}_{k}\right)_{k},\left(\mathbf{C}_{j}^{*}\right)_{j},\left(\mathbf{R}_{k}^{*}\right)_{k}$

$\in \ell^{\infty}\left(\mathbb{N}, \ell_{\text {SOT }}^{2}(\mathbb{N}, \mathcal{B}(H))\right)$.

Proof. Since for each $\mathbf{y} \in \ell^{2}(H), x, y \in H$ and $k, j \in \mathbb{N}$ we have

$$
\left\langle\left\langle\mathbf{A}\left(x \mathbf{e}_{k}\right), \mathbf{y}\right\rangle\right\rangle=\left\langle\left\langle\mathbf{R}_{k}(x), \mathbf{y}\right\rangle\right\rangle
$$

and

$$
\left\langle\left\langle\mathbf{A}(\mathbf{x}), y \mathbf{e}_{j}\right\rangle\right\rangle=\left\langle\left\langle\mathbf{x}, \mathbf{C}_{j}(y)\right\rangle\right\rangle
$$

we clearly have

$$
\left\|\mathbf{R}_{k}\right\|_{\ell_{\mathrm{SOT}}^{2}}(\mathbb{N}, \mathcal{B}(H))=\sup _{\|x\|=1} \sup _{\|\mathbf{y}\|_{\ell^{2}(H)}=1} \mid\left\langle\left\langle\mathbf{A}\left(x \mathbf{e}_{k}\right), \mathbf{y}\right\rangle\right| \leq\|\mathbf{A}\| .
$$

A similar argument allows to obtain $\left\|\mathbf{C}_{j}\right\|_{\ell_{\text {SOT }}^{2}(\mathbb{N}, \mathcal{B}(H))} \leq\|\mathbf{A}\|$. Now, since $\left\|T_{k j}\right\|=\left\|T_{k j}^{*}\right\|$, applying the fact that rows in $\mathbf{A}^{*}$ correspond with the adjoint operators in the columns in $\mathbf{A}$ we obtain the other cases.

Let us give another necessary condition for boundedness to be used later on.

Proposition 4.8. Let $\mathbf{A}=\left(T_{k j}\right) \in \mathcal{B}\left(\ell^{2}(H)\right)$. Then

$$
\sum_{k=1}^{\infty} \sum_{j=1}^{\infty}\left\|T_{k j} x_{j}\right\|^{2} \leq\|\mathbf{A}\|^{2} \sum_{j=1}^{\infty}\left\|x_{j}\right\|^{2} .
$$


Proof. Let $\mathbf{x} \in \ell^{2}(H)$ and assume that $\sum_{j=1}^{\infty}\left\|x_{j}\right\|^{2}=1$. Denote by $F_{\mathbf{x}}:[0,2 \pi] \rightarrow$ $\ell^{2}(H)$ the continuous function given by $F_{\mathbf{x}}(s)=\left(x_{j} \varphi_{j}(s)\right)$. Trivially, we have $\|\mathbf{x}\|=$ $\left\|F_{\mathbf{x}}\right\|_{C\left(\mathbb{T}, \ell^{2}(H)\right)}$. Then

$$
\begin{aligned}
\sum_{k=1}^{\infty} \sum_{j=1}^{\infty}\left\|T_{k j} x_{j}\right\|^{2} & =\sum_{k=1}^{\infty} \int_{0}^{2 \pi}\left\|\sum_{j=1}^{\infty} T_{k j} x_{j} \varphi_{j}(s)\right\|^{2} \frac{d s}{2 \pi} \\
& =\int_{0}^{2 \pi} \sum_{k=1}^{\infty}\left\|\sum_{j=1}^{\infty} T_{k j} x_{j} \varphi_{j}(s)\right\|^{2} \frac{d s}{2 \pi} \\
& =\int_{0}^{2 \pi}\left\|\mathbf{A}\left(F_{\mathbf{x}}(s)\right)\right\|^{2} \frac{d s}{2 \pi} \\
& \leq\|\mathbf{A}\|^{2} \int_{0}^{2 \pi}\left\|F_{\mathbf{x}}(s)\right\|^{2} \frac{d s}{2 \pi}=\|\mathbf{A}\|^{2} .
\end{aligned}
$$

This concludes the result.

From Proposition 4.8 we can get an extension of Schur theorem to matrices whose entries are operators in $\mathcal{B}(H)$.

Theorem 4.9. Let $\mathbf{A}=\left(T_{k j}\right)$ and $\mathbf{B}=\left(S_{k j}\right)$. If $\mathbf{A}, \mathbf{B} \in \mathcal{B}\left(\ell^{2}(H)\right)$ then $\mathbf{A} * \mathbf{B} \in \mathcal{B}\left(\ell^{2}(H)\right)$. Moreover

$$
\|\mathbf{A} * \mathbf{B}\|_{\mathcal{B}\left(\ell^{2}(H)\right)} \leq\|\mathbf{A}\|_{\mathcal{B}\left(\ell^{2}(H)\right)}\|\mathbf{B}\|_{\mathcal{B}\left(\ell^{2}(H)\right)} .
$$

Proof. It suffices to show that if $\mathbf{x}, \mathbf{y} \in c_{00}(H)$ then

$$
|\langle\mathbf{A} * \mathbf{B}(\mathbf{x}), \mathbf{y}\rangle| \mid \leq\|\mathbf{A}\|\|\mathbf{B}\|\|\mathbf{x}\|\|\mathbf{y}\|
$$

Notice that

$$
\begin{aligned}
|\langle\mathbf{A} * \mathbf{B}(\mathbf{x}), \mathbf{y}\rangle\rangle \mid & =\left|\sum_{k=1}^{\infty}\left\langle\sum_{j=1}^{\infty} T_{k j} S_{k j}\left(x_{j}\right), y_{k}\right\rangle\right| \\
& =\left|\sum_{k=1}^{\infty} \sum_{j=1}^{\infty}\left\langle S_{k j}\left(x_{j}\right), T_{k j}^{*}\left(y_{k}\right)\right\rangle\right| \\
& \leq \sum_{k=1}^{\infty} \sum_{j=1}^{\infty}\left\|T_{k j}^{*}\left(y_{k}\right)\right\|\left\|S_{k j}\left(x_{j}\right)\right\| \\
& \leq\left(\sum_{k=1}^{\infty} \sum_{j=1}^{\infty}\left\|T_{k j}^{*}\left(y_{k}\right)\right\|^{2}\right)^{1 / 2}\left(\sum_{k=1}^{\infty} \sum_{j=1}^{\infty}\left\|S_{k j}\left(x_{j}\right)\right\|^{2}\right)^{1 / 2} .
\end{aligned}
$$

Using the estimate above and applying Proposition 4.8 to $\mathbf{B}$ and $\mathbf{A}^{*}$, we obtain 4.2 immediately since $\|\mathbf{A}\|=\left\|\mathbf{A}^{*}\right\|$. The proof is then complete. 
Given $S \subset \mathbb{N} \times \mathbb{N}$ and $\mathbf{A}=\left(T_{k j}\right)$, we write $P_{S} \mathbf{A}=\left(T_{k j} \chi_{S}\right)$, that is the matrix with entries $T_{k j}$ if $(k, j) \in S$ and 0 otherwise. In particular, matrices with a single row, column or diagonal correspond to $S=\{k\} \times \mathbb{N}, S=\mathbb{N} \times\{j\}$ and $D_{l}=\{(k, k+l): k \in \mathbb{N}\}$ for $l \in \mathbb{Z}$ respectively. Also, the case of finite or upper (or lower) triangular matrices coincides with $P_{S} \mathbf{A}$ for $S=[1, N] \times[1, M]=\{(k, j): 1 \leq k \leq N, 1 \leq j \leq M\}$ or $S=\Delta=\{(k, j): j \geq k\}$ (or $S=\{(k, j): j \leq k\}$ ) respectively.

It is well known that the mapping $\mathbf{A} \rightarrow P_{S} \mathbf{A}$ is not continuous in $\mathcal{B}(H)$ for all sets $S$ (for instance, the reader is referred to [10. Chapter 2, Theorem 2.19] to see that $S=\Delta$ the triangle projection is unbounded) but there are cases where this holds true. Clearly we have that $\mathbf{A} \in \mathcal{B}\left(\ell^{2}(H)\right)$ if and only if $\|\mathbf{A}\|=\sup _{N, M}\left\|P_{[1, N] \times[1, M]} \mathbf{A}\right\|<\infty$. This easily follows noticing that

$$
\left\langle\left\langle P_{[1, N] \times[1, M]} \mathbf{A}(\mathbf{x}), \mathbf{y}\right\rangle\right\rangle=\left\langle\left\langle\mathbf{A}\left(P_{N} \mathbf{x}\right), P_{M} \mathbf{y}\right\rangle\right\rangle
$$

where $P_{N} \mathbf{x}$ stands for the projection on the $N$-first coordinates of $\mathbf{x}$.

In general it is rather difficult to compute the norm of the matrix $\mathbf{A}$. Let us consider some trivial cases.

Corollary 4.10. Let $\mathbf{A}=\left(T_{k j}\right) \subset \mathcal{B}(H)$. Then

(i) $\left\|P_{\mathbb{N} \times\{j\}} \mathbf{A}\right\|=\left\|\mathbf{C}_{j}\right\|_{\ell_{\mathrm{SOT}}^{2}(\mathbb{N}, \mathcal{B}(H))}$ for each $j \in \mathbb{N}$.

(ii) $\left\|P_{\{k\} \times \mathbb{N}} \mathbf{A}\right\|=\left\|\mathbf{R}_{k}\right\|_{\ell_{\mathrm{SOT}}^{2}}(\mathbb{N}, \mathcal{B}(H))$ for each $k \in \mathbb{N}$.

(iii) $\left\|P_{D_{l}} \mathbf{A}\right\|=\sup _{k}\left\|T_{k, k+l}\right\|$ for each $l \in \mathbb{Z}$ (where $T_{k, k+l}=0$ whenever $\left.k+l \leq 0\right)$.

Proof. (i) and (ii) follow trivially from Lemma 4.7 .

To see (iii), note that $\left(P_{D_{l}} \mathbf{A}(\mathbf{x})\right)_{k}=\left(T_{k, k+l} x_{k+l}\right)_{k}$. Hence $\left\|P_{D_{l}} \mathbf{A}(\mathbf{x})\right\| \leq$ $\left(\sup _{k}\left\|T_{k, k+l}\right\|\right)\|\mathbf{x}\|$. Since the other inequality always holds, the proof is complete.

\section{Toeplitz multipliers on operator-valued matrices}

In this section we shall achieve the operator-valued analogues to the Toeplitz and Bennet theorems presented in the introduction.

Theorem 5.1. Let $\mathbf{A}=\left(T_{k j}\right) \in \mathcal{T}$. Then $A \in \mathcal{B}\left(\ell^{2}(H)\right)$ if and only if there exists $\mu \in V^{\infty}(\mathbb{T}, \mathcal{B}(H))$ such that $T_{k j}=\widehat{\mu}(j-k)$ for all $k, j \in \mathbb{N}$. Moreover, $\|\mathbf{A}\|=\|\mu\|_{\infty}$.

Proof. Assume that $\mu \in V^{\infty}(\mathbb{T}, \mathcal{B}(H))$ and $T_{k j}=\widehat{\mu}(j-k)$ for all $k, j \in \mathbb{N}$. Then, for 
$\mathbf{x}, \mathbf{y} \in c_{00}(H)$, we have

$$
\begin{aligned}
\langle\langle\mathbf{A}(\mathbf{x}), \mathbf{y}\rangle\rangle & =\sum_{k=1}^{M} \sum_{j=1}^{N}\left\langle T_{k j}\left(x_{j}\right), y_{k}\right\rangle=\sum_{k=1}^{M} \sum_{j=1}^{N}\left\langle T_{\mu}\left(\overline{\varphi_{j}} \varphi_{k}\right)\left(x_{j}\right), y_{k}\right\rangle \\
& =\sum_{k=1}^{M} \sum_{j=1}^{N} \Psi_{\mu}\left(\bar{\varphi}_{j} x_{j} \otimes \bar{\varphi}_{k} y_{k}\right)=\Psi_{\mu}\left(\sum_{k=1}^{M} \sum_{j=1}^{N} \bar{\varphi}_{j} x_{j} \otimes \bar{\varphi}_{k} y_{k}\right) \\
& =\Psi_{\mu}\left(\left(\sum_{j=1}^{N} \bar{\varphi}_{j} x_{j}\right) \otimes\left(\sum_{k=1}^{M} \bar{\varphi}_{k} y_{k}\right)\right) .
\end{aligned}
$$

Therefore

$$
\begin{aligned}
|\langle\mathbf{A}(\mathbf{x}), \mathbf{y}\rangle\rangle \mid & \left.\leq\left\|\Psi_{\mu}\right\|_{L^{1}(\mathbb{T}, H \widehat{\otimes} H)^{*}} \int_{0}^{2 \pi} \| h_{\mathbf{x}}(-t) \otimes h_{\mathbf{y}}(-t)\right) \|_{H \widehat{\otimes} H} \frac{d t}{2 \pi} \\
& =\|\mu\|_{\infty} \int_{0}^{2 \pi}\left\|h_{\mathbf{x}}(-t)\right\|\left\|h_{\mathbf{y}}(-t)\right\| \frac{d t}{2 \pi} \\
& \left.\leq\|\mu\|_{\infty}\left(\int_{0}^{2 \pi}\left\|h_{\mathbf{x}}(-t)\right\|^{2} \frac{d t}{2 \pi}\right)^{1 / 2}\left(\int_{0}^{2 \pi} \| h_{\mathbf{y}}(-t)\right) \|^{2} \frac{d t}{2 \pi}\right)^{1 / 2} \\
& \leq\|\mu\|_{\infty}\|\mathbf{x}\|_{\ell^{2}(H)}\|\mathbf{y}\|_{\ell^{2}(H)} .
\end{aligned}
$$

Hence, $\mathbf{A} \in \mathcal{B}\left(\ell^{2}(H)\right)$ and $\|\mathbf{A}\| \leq\|\mu\|_{\infty}$.

Conversely, let us assume that $\mathbf{A} \in \mathcal{B}\left(\ell^{2}(H)\right)$ and $T_{k j}=T_{j-k}$ for a given sequence $\mathbf{T}=\left(T_{n}\right)_{n \in \mathbb{Z}}$ of operators in $\mathcal{B}(H)$. We define

$$
T\left(\sum_{n=-M}^{N} \alpha_{n} \varphi_{n}\right)=\alpha_{0} T_{1,1}+\sum_{n=1}^{M} \alpha_{-n} T_{n+1,1}+\sum_{n=1}^{N} \alpha_{n} T_{1, n+1}
$$

We are going to show that $T \in \mathcal{L}\left(L^{1}(\mathbb{T}), \mathcal{B}(H)\right)$. Since $L^{1}(\mathbb{T})=\overline{\operatorname{span}\left\{\varphi_{k}: k \in \mathbb{Z}\right\}} \|^{\|\cdot\|_{1}}$, it suffices to show that

$$
\left\|T\left(\sum_{n=-M}^{N} \alpha_{n} \varphi_{n}\right)\right\| \leq\|\mathbf{A}\| \int_{0}^{2 \pi}\left|\sum_{n=-M}^{N} \alpha_{n} \varphi_{n}(t)\right| \frac{d t}{2 \pi} .
$$

Let $x, y \in H$ and notice that

$$
\left\langle T\left(\sum_{n=-M}^{N} \alpha_{n} \varphi_{n}\right)(x), y\right\rangle=\sum_{n=-M}^{N} \alpha_{n} \beta_{n}(x, y),
$$

where $\beta_{n}(x, y)=\left\langle T_{n}(x), y\right\rangle$. Now taking into account that $A_{x, y}=\left(\left\langle T_{k j}(x), y\right\rangle\right)$ is a Toeplitz matrix and defines a bounded operator $A_{x, y} \in \mathcal{B}\left(\ell^{2}\right)$ with $\left\|A_{x, y}\right\| \leq\|\mathbf{A}\|\|x\|\|y\|$ we obtain, due to Theorem 1.2 , that

$$
\psi_{x, y}=\sum_{n \in \mathbb{Z}} \beta_{n}(x, y) \varphi_{n} \in L^{\infty}(\mathbb{T})
$$


with $\left\|\psi_{x, y}\right\|_{L^{\infty}(\mathbb{T})} \leq\|\mathbf{A}\|\|x\|\|y\|$. Finally, we have

$$
\begin{aligned}
\left|\left\langle T\left(\sum_{n=-M}^{N} \alpha_{n} \varphi_{n}\right)(x), y\right\rangle\right| & =\left|\int_{0}^{2 \pi}\left(\sum_{n=-M}^{N} \alpha_{n} \varphi_{n}(t)\right) \overline{\psi_{x, y}(t)} \frac{d t}{2 \pi}\right| \\
& \leq\left\|\sum_{n=-M}^{N} \alpha_{n} \varphi_{n}(t)\right\|_{L^{1}(\mathbb{T})}\|\mathbf{A}\|\|x\|\|y\| .
\end{aligned}
$$

This shows (5.1) which gives $\|T\|_{L^{1}(\mathbb{T}) \rightarrow \mathcal{B}(H)} \leq\|\mathbf{A}\|$. Finally, from the embedding $C(\mathbb{T}) \rightarrow$ $L^{1}(\mathbb{T})$ we have that there exists $\mu \in V^{\infty}(\mathbb{T}, \mathcal{B}(H))$ such that $T_{\mu}=T$ and $\|\mu\|_{\infty} \leq\|A\|$. The proof is then complete.

To prove the analogue of Bennet't theorem on Schur multipliers we shall need the following lemmas.

Lemma 5.2. Let $\mathbf{A}=\left(T_{k j}\right) \in \mathcal{M}_{l}\left(\ell^{2}(H)\right) \cup \mathcal{M}_{r}\left(\ell^{2}(H)\right)$ and $x_{0}, y_{0} \in H$ with $\left\|x_{0}\right\|=$ $\left\|y_{0}\right\|=1$. Denote by $A_{x_{0}, y_{0}}=\left(\gamma_{k j}\right)$ the matrix with entries

$$
\gamma_{k j}=\left\langle T_{k j}\left(x_{0}\right), y_{0}\right\rangle, \quad k, j \in \mathbb{N} .
$$

Then $A_{x_{0}, y_{0}} \in \mathcal{M}\left(\ell^{2}\right)$ and $\left\|A_{x_{0}, y_{0}}\right\|_{\mathcal{M}\left(\ell^{2}\right)} \leq \min \left\{\|\mathbf{A}\|_{\mathcal{M}_{l}\left(\ell^{2}(H)\right)},\|\mathbf{A}\|_{\mathcal{M}_{r}\left(\ell^{2}(H)\right)}\right\}$.

Proof. Let $z_{0} \in H$ and $\left\|z_{0}\right\|=1$ and consider the bounded operators $\pi_{z_{0}}: \ell^{2}(H) \rightarrow \ell^{2}$ and $i_{z_{0}}: \ell^{2} \rightarrow \ell^{2}(H)$ given by

$$
\pi_{z_{0}}\left(\left(x_{j}\right)\right)=\left(\left\langle x_{j}, z_{0}\right\rangle\right)_{j}, \quad i_{z_{0}}\left(\left(\alpha_{k}\right)\right)=\left(\alpha_{k} z_{0}\right)_{k} .
$$

Now, given $B=\left(\beta_{k j}\right) \in \mathcal{B}\left(\ell^{2}\right)$ with $\|B\|=1$, we define $\mathbf{B}=i_{z_{0}} B \pi_{z_{0}}$.

Hence $\mathbf{B} \in \mathcal{B}\left(\ell^{2}(H)\right)$. Moreover $\|\mathbf{B}\|=\|B\|$ because $\left\|i_{z_{0}}\right\|=\left\|\pi_{z_{0}}\right\|=1$ and $B\left(\left(\alpha_{j}\right)\right) z_{0}=$ $\mathbf{B}\left(\left(\alpha_{j} z_{0}\right)\right)$ for any $\left(\alpha_{j}\right) \in \ell^{2}$.

Let us write $\mathbf{B}=\left(S_{k j}\right)$ and observe that $S_{k j}=\beta_{k j} \widetilde{z_{0} \otimes z_{0}}$. Indeed,

$$
\left\langle S_{k j}(x), y\right\rangle=\left\langle\left\langle\mathbf{B}\left(x \mathbf{e}_{j}\right), y \mathbf{e}_{k}\right\rangle\right\rangle=\left\langle\left\langle\left(\left\langle x, z_{0}\right\rangle \beta_{k j} z_{0}\right)_{k}, y \mathbf{e}_{k}\right\rangle\right\rangle=\beta_{k j}\left\langle x, z_{0}\right\rangle\left\langle z_{0}, y\right\rangle .
$$

Recall that $T(\widetilde{x \otimes y})=x \widetilde{\otimes T(y)}$ and $\widetilde{(x \otimes y}) T=\widetilde{T^{*} x \otimes y}$ for any $T \in \mathcal{B}(H)$ and $x, y \in H$. In particular we obtain

$$
\left\langle\left(T_{k j} S_{k j}\right)\left(x_{0}\right), y_{0}\right\rangle=\beta_{k j}\left\langle T_{k j}\left(z_{0}\right), y_{0}\right\rangle\left\langle x_{0}, z_{0}\right\rangle
$$

and

$$
\left\langle\left(S_{k j} T_{k j}\right)\left(x_{0}\right), y_{0}\right\rangle=\beta_{k j}\left\langle T_{k j}\left(x_{0}\right), z_{0}\right\rangle\left\langle z_{0}, y_{0}\right\rangle \text {. }
$$

Therefore, choosing $z_{0}=x_{0}$ and $\mathbf{C}=\mathbf{A} * \mathbf{B}$ one has $C_{x_{0}, y_{0}}=A_{x_{0}, y_{0}} * B$, and using that $\left\|C_{x_{0}, y_{0}}\right\| \leq\|\mathbf{C}\|$ we obtain

$$
\left\|A_{x_{0}, y_{0}} * B\right\|_{\mathcal{B}\left(\ell^{2}\right)} \leq\|\mathbf{A} * \mathbf{B}\|_{\mathcal{B}\left(\ell^{2}(H)\right)} \leq\|\mathbf{A}\|_{\mathcal{M}_{l}\left(\ell^{2}(H)\right)}
$$


Similarly, choosing $z_{0}=y_{0}$ and $\mathbf{C}=\mathbf{B} * \mathbf{A}$ one obtains

$$
\left\|B * A_{x_{0}, y_{0}}\right\|_{\mathcal{B}\left(\ell^{2}\right)} \leq\|\mathbf{A}\|_{\mathcal{M}_{r}\left(\ell^{2}(H)\right)} .
$$

This completes the proof.

Lemma 5.3. Let $\mu \in \mathfrak{M}(\mathbb{T}, \mathcal{B}(H)), \mathbf{A}=\left(T_{k j}\right) \in \mathcal{T}$ with $T_{k j}=\widehat{\mu}(j-k)$ for $k, j \in \mathbb{N}$, $\mathbf{B}=\left(S_{k j}\right) \subset \mathcal{B}(H)$ and $\mathbf{x}, \mathbf{y} \in c_{00}(H)$. Then

$$
\langle\langle\mathbf{A} * \mathbf{B}(\mathbf{x}), \mathbf{y}\rangle\rangle=\Psi_{\mu}\left(\int_{0}^{2 \pi} \int_{0}^{2 \pi} \mathbf{B}_{N, M}(s-\cdot, t-\cdot)\left(h_{\mathbf{x}}(s)\right) \otimes h_{\mathbf{y}}(t) \frac{d s}{2 \pi} \frac{d t}{2 \pi}\right) .
$$

Proof. Let $\mathbf{x}, \mathbf{y} \in c_{00}(H)$, say $h_{\mathbf{x}}=\sum_{j=1}^{N} x_{j} \varphi_{j}$ and $h_{\mathbf{y}}=\sum_{k=1}^{M} y_{k} \varphi_{k}$. Recall that $x_{j}=$ $\int_{0}^{2 \pi} h_{\mathbf{x}}(s) \overline{\varphi_{j}(s)} \frac{d s}{2 \pi}$ and $y_{k}=\int_{0}^{2 \pi} h_{\mathbf{y}}(t) \overline{\varphi_{k}(t)} \frac{d t}{2 \pi}$. Then

$$
\begin{aligned}
& \langle\langle\mathbf{A} * \mathbf{B}(\mathbf{x}), \mathbf{y}\rangle\rangle \\
& =\sum_{k=1}^{M} \sum_{j=1}^{N}\left\langle\widehat{\mu}(j-k) S_{k j}\left(x_{j}\right), y_{k}\right\rangle \\
& =\int_{0}^{2 \pi}\left\langle\sum_{k=1}^{M}\left(\sum_{j=1}^{N} \widehat{\mu}(j-k) S_{k j}\left(x_{j}\right)\right) \varphi_{k}(t), h_{\mathbf{y}}(t)\right\rangle \frac{d t}{2 \pi} \\
& =\int_{0}^{2 \pi}\left\langle\sum_{l=-M}^{N} \widehat{\mu}(l)\left(\sum_{j-k=l} S_{k j}\left(x_{j}\right) \varphi_{k}(t)\right), h_{\mathbf{y}}(t)\right\rangle \frac{d t}{2 \pi} \\
& =\int_{0}^{2 \pi} \int_{0}^{2 \pi}\left\langle\sum_{l=-M}^{N} \widehat{\mu}(l)\left(\sum_{j-k=l} S_{k j} \overline{\varphi_{j}(s)} \varphi_{k}(t)\left(h_{\mathbf{x}}(s)\right)\right), h_{\mathbf{y}}(t)\right\rangle \frac{d s}{2 \pi} \frac{d t}{2 \pi} \\
& =\int_{0}^{2 \pi} \int_{0}^{2 \pi} \sum_{l=-M}^{N} \mathcal{J} \mu(l)\left(\left(\sum_{j-k=l} S_{k j} \overline{\varphi_{j}(s)} \varphi_{k}(t)\right)\left(h_{\mathbf{x}}(s)\right) \otimes h_{\mathbf{y}}(t)\right) \frac{d s}{2 \pi} \frac{d t}{2 \pi} \\
& =\sum_{l=-M}^{N} \mathcal{J} \mu(l)\left(\int_{0}^{2 \pi} \int_{0}^{2 \pi}\left(\sum_{j-k=l} S_{k j} \overline{\varphi_{j}(s)} \varphi_{k}(t)\right)\left(h_{\mathbf{x}}(s)\right) \otimes h_{\mathbf{y}}(t) \frac{d s}{2 \pi} \frac{d t}{2 \pi}\right) \\
& =\Psi_{\mu}\left(\sum_{l=-M}^{N}\left(\int_{0}^{2 \pi} \int_{0}^{2 \pi}\left(\sum_{j-k=l} S_{k j} \overline{\varphi_{j}(s)} \varphi_{k}(t)\right)\left(h_{\mathbf{x}}(s)\right) \otimes h_{\mathbf{y}}(t) \frac{d s}{2 \pi} \frac{d t}{2 \pi}\right) \varphi_{l}\right) \\
& =\Psi_{\mu}\left(\int_{0}^{2 \pi} \int_{0}^{2 \pi}\left(\sum_{k=1}^{M} \sum_{j=l}^{N} S_{k j} \overline{\varphi_{j}(s)} \varphi_{k}(t) \varphi_{j} \varphi_{-k}\right)\left(h_{\mathbf{x}}(s)\right) \otimes h_{\mathbf{y}}(t) \frac{d s}{2 \pi} \frac{d t}{2 \pi}\right) \\
& =\Psi_{\mu}\left(\int_{0}^{2 \pi} \int_{0}^{2 \pi} \mathbf{B}_{N, M}(s-\cdot, t-\cdot)\left(h_{\mathbf{x}}(s)\right) \otimes h_{\mathbf{y}}(t) \frac{d t}{2 \pi} \frac{d s}{2 \pi}\right) .
\end{aligned}
$$

The proof is complete. 
Theorem 5.4. If $\mu \in M(\mathbb{T}, \mathcal{B}(H))$ and $\mathbf{A}=\left(T_{k j}\right) \in \mathcal{T}$ with $T_{k j}=\widehat{\mu}(j-k)$ for $k, j \in \mathbb{N}$ then $\mathbf{A} \in \mathcal{M}_{l}\left(\ell^{2}(H)\right) \cap \mathcal{M}_{r}\left(\ell^{2}(H)\right)$ and

$$
\max \left\{\|\mathbf{A}\|_{\mathcal{M}_{l}\left(\ell^{2}(H)\right)},\|\mathbf{A}\|_{\mathcal{M}_{r}\left(\ell^{2}(H)\right)}\right\} \leq|\mu|
$$

Proof. Since $\|\mathbf{A}\|_{\mathcal{M}_{l}\left(\ell^{2}(H)\right)}=\left\|\mathbf{A}^{*}\right\|_{\mathcal{M}_{l}\left(\ell^{2}(H)\right)}$ and $|\mu|=\left|\mu^{*}\right|$ then it suffices to show the case of left Schur multipliers. Let $\mathbf{x}, \mathbf{y} \in c_{00}(H)$ and $\mathbf{B}=\left(S_{k j}\right) \subset \mathcal{B}(H)$ such that $\mathbf{B} \in \mathcal{B}\left(\ell^{2}(H)\right)$. Define

$$
G(u)=\int_{0}^{2 \pi} \int_{0}^{2 \pi} \mathbf{B}_{N, M}(s-u, t-u)\left(h_{\mathbf{x}}(s)\right) \otimes h_{\mathbf{y}}(t) \frac{d t}{2 \pi} \frac{d s}{2 \pi} .
$$

Hence we can rewrite, since $(\lambda x) \otimes y=x \otimes \bar{\lambda} y$,

$$
G(u)=\sum_{k=1}^{\infty} \sum_{j=1}^{\infty} S_{k j}\left(x_{j} \varphi_{j}(u)\right) \otimes y_{k} \varphi_{k}(u)
$$

In particular,

$$
\begin{aligned}
\|G(u)\|_{H \widehat{\otimes} H} & \leq \sum_{k=1}^{\infty}\left\|\sum_{j=1}^{\infty} S_{k j}\left(x_{j} \varphi_{j}(u)\right)\right\|\left\|y_{k} \varphi_{k}(u)\right\| \leq\left(\sum_{k=1}^{\infty}\left\|\sum_{j=1}^{\infty} S_{k j}\left(x_{j} \varphi_{j}(u)\right)\right\|^{2}\right)^{1 / 2}\|\mathbf{y}\| \\
& \leq\|\mathbf{B}\|\|\mathbf{x}\|\|\mathbf{y}\| .
\end{aligned}
$$

From Lemma 5.3, we have

$$
|\langle\langle\mathbf{A} * \mathbf{B}(\mathbf{x}), \mathbf{y}\rangle\rangle| \leq\left\|\Psi_{\mu}\right\|_{C(\mathbb{T}, H \widehat{\otimes} H)^{*}} \sup _{0 \leq u<2 \pi}\|G(u)\|_{H \widehat{\otimes} H}=|\mu|\|\mathbf{B}\|\|\mathbf{x}\|\|\mathbf{y}\| .
$$

This finishes the proof.

Lemma 5.5. Let $\mu, \nu \in \mathfrak{M}(\mathbb{T}, \mathcal{B}(H)), \mathbf{A}=\left(T_{k j}\right) \in \mathcal{T}$ with $T_{k j}=\widehat{\mu}(j-k), \mathbf{B}=\left(S_{k j}\right) \in \mathcal{T}$ with $S_{k j}=\widehat{\nu}(j-k)$ for $k, j \in \mathbb{N}$ and $\mathbf{x}, \mathbf{y} \in c_{00}(H)$. Then

$$
\langle\langle\mathbf{A} * \mathbf{B}(\mathbf{x}), \mathbf{y}\rangle\rangle=\Psi_{\mu}\left(\sum_{k=1}^{M}\left(\sum_{j=1}^{N} \widehat{\nu}(j-k)\left(x_{j}\right) \overline{\varphi_{j}}\right) \otimes y_{k} \varphi_{k}\right) .
$$

Proof. Denote $h_{\mathbf{x}}=\sum_{k=1}^{M} y_{k} \varphi_{k}$ and $h_{\mathbf{y}}=\sum_{j=1}^{N} x_{j} \varphi_{j}$. Then

$$
\begin{aligned}
\langle\langle\mathbf{A} * \mathbf{B}(\mathbf{x}), \mathbf{y}\rangle\rangle & =\sum_{k=1}^{M} \sum_{j=1}^{N}\left\langle\widehat{\mu}(j-k) \widehat{\nu}(j-k)\left(x_{j}\right), y_{k}\right\rangle=\sum_{l=-M}^{N} \sum_{k=1}^{M}\left\langle\widehat{\mu}(l) \widehat{\nu}(l)\left(x_{k+l}\right), y_{k}\right\rangle \\
& =\sum_{l=-M}^{N} \sum_{k=1}^{M} \mathcal{J} \widehat{\mu}(l)\left(\nu(l)\left(x_{k+l}\right) \otimes y_{k}\right)=\sum_{l=-M}^{N} \mathcal{J} \widehat{\mu}(l)\left(\sum_{k=1}^{M} \widehat{\nu}(l)\left(x_{k+l}\right) \otimes y_{k}\right)
\end{aligned}
$$




$$
\begin{aligned}
& =\Psi_{\mu}\left(\sum_{l=-M}^{N}\left(\sum_{k=1}^{M} \widehat{\nu}(l)\left(x_{k+l}\right) \otimes y_{k}\right) \varphi_{-l}\right) \\
& =\Psi_{\mu}\left(\sum_{k=1}^{M}\left(\sum_{j=1}^{N} \widehat{\nu}(j-k)\left(x_{j}\right) \overline{\varphi_{j}}\right) \otimes y_{k} \overline{\varphi_{k}}\right) .
\end{aligned}
$$

The proof is complete.

Corollary 5.6. Let $\mathbf{A}=\left(S_{k j}\right) \in \mathcal{T}$ such that $S_{k j}=\widehat{\nu}(j-k)$ for some $\nu \in \mathfrak{M}(T, \mathcal{B}(H))$. For each $\mathbf{x}, \mathbf{y} \in c_{00}(H)$ we denote

$$
F_{\mathbf{x}, \mathbf{y}, \mathbf{A}}(t)=\sum_{k=1}^{\infty}\left(\sum_{j=1}^{\infty} \widehat{\nu}(j-k)\left(x_{j}\right) \overline{\varphi_{j}}(t)\right) \otimes y_{k} \overline{\varphi_{k}}(t) .
$$

If $\mathbf{A} \in \mathcal{M}_{r}\left(\ell^{2}(H)\right)$ then

$$
\left\|F_{\mathbf{x}, \mathbf{y}, \mathbf{A}}\right\|_{L^{1}(\mathbb{T}, H \widehat{\otimes} H)} \leq\|\mathbf{A}\|_{\mathcal{M}_{r}\left(\ell^{2}(H)\right)}\|\mathbf{x}\|_{\ell^{2}(H)}\|\mathbf{y}\|_{\ell^{2}(H)} .
$$

Proof. If $\mathbf{A} \in \mathcal{M}_{r}\left(\ell^{2}(H)\right)$ then $\mathbf{B} * \mathbf{A} \in \mathcal{B}\left(\ell^{2}(H)\right)$ for any $\mathbf{B} \in \mathcal{B}\left(\ell^{2}(H)\right) \cap \mathcal{T}$. In particular for any $\mathbf{B}=\left(T_{k j}\right)$ with $T_{k j}=\widehat{\mu}(j-k)$ for some $\mu \in V^{\infty}(\mathbb{T}, \mathcal{B}(H))$ with $\|\mu\|_{\infty}=\|\mathbf{B}\|$. Since $L^{1}(\mathbb{T}, H \widehat{\otimes} H) \subseteq\left(V^{\infty}(\mathbb{T}, B(H))\right)^{*}$ isometrically, we can use Lemma 5.5 to obtain

$$
\begin{aligned}
\left\|F_{\mathbf{x}, \mathbf{y}, \mathbf{A}}\right\|_{L^{1}(\mathbb{T}, H \widehat{\otimes} H)} & =\sup \left\{\left|\Psi_{\mu}\left(F_{\mathbf{x}, \mathbf{y}, \mathbf{A}}\right)\right|:\|\mu\|_{\infty}=1\right\} \\
& =\sup \{|\langle\langle\mathbf{B} * \mathbf{A}(\mathbf{x}), \mathbf{y}\rangle\rangle|:\|\mathbf{B}\|=1\} \\
& \leq\|\mathbf{A}\|_{\mathcal{M}_{r}\left(\ell^{2}(H)\right)}\|\mathbf{x}\|_{\ell^{2}(H)}\|\mathbf{y}\|_{\ell^{2}(H)} .
\end{aligned}
$$

This completes the proof.

Theorem 5.7. Let $\mathbf{A}=\left(T_{k j}\right) \in \mathcal{T} \cap \mathcal{M}_{r}\left(\ell^{2}(H)\right)$. Then there exists $\mu \in M_{\mathrm{SOT}}(\mathbb{T}, \mathcal{B}(H))$ such that $T_{k j}=\widehat{\mu}(j-k)$ for all $k, j \in \mathbb{N}$. Moreover, $\|\mu\|_{\mathrm{SOT}} \leq\|\mathbf{A}\|_{\mathcal{M}_{r}\left(\ell^{2}(H)\right)}$.

Proof. Let $\mathbf{A} \in \mathcal{M}_{r}\left(\ell^{2}(H)\right)$. For each $x_{0}, y_{0} \in H$, as above we consider the scalar-valued Toeplitz matrix $A_{x_{0}, y_{0}}=\left(\left\langle T_{k j}\left(x_{0}\right), y_{0}\right\rangle\right)$. Using Lemma 5.2, we have that $A_{x_{0}, y_{0}} \in \mathcal{M}\left(\ell^{2}\right)$ and $\left\|A_{x_{0}, y_{0}}\right\|_{\mathcal{M}\left(\ell^{2}\right)} \leq\|\mathbf{A}\|_{\mathcal{M}\left(\ell^{2}(H)\right)}$. This guarantees, invoking Theorem 1.3 , that there exists $\eta_{x_{0}, y_{0}} \in M(\mathbb{T})$ such that $\left\langle T_{k j}\left(x_{0}\right), y_{0}\right\rangle=\widehat{\eta_{x_{0}, y_{0}}}(j-k)$ for all $j, k \in \mathbb{N}$ and $\left|\eta_{x_{0}, y_{0}}\right|=$ $\left\|A_{x_{0}, y_{0}}\right\|_{\mathcal{M}_{r}\left(\ell^{2}\right)}$.

Now define $\mu(A) \in \mathcal{B}(H)$ given by

$$
\langle\mu(A)(x), y\rangle=\eta_{x, y}(A), \quad x, y \in H
$$

Let us show that $\mu \in M_{\mathrm{SOT}}(\mathbb{T}, \mathcal{B}(H))$ and $\|\mu\|_{\mathrm{SOT}} \leq\|\mathbf{A}\|_{\mathcal{M}_{r}\left(\ell^{2}(H)\right)}$. 
First we need to show that $\mu(A) \in \mathcal{B}(H)$ for any $A \in \mathfrak{B}(\mathbb{T})$. This follows using that

$$
\eta_{\lambda x+\beta x^{\prime}, y}(l)=\lambda \widehat{\eta_{x, y}}(l)+\beta \widehat{\eta_{x^{\prime}, y}}(l), \quad l \in \mathbb{Z}
$$

for any $\lambda, \beta \in \mathbb{C}$ and $x, x^{\prime}, y \in H$. This guarantees that $\eta_{\lambda x+\beta x^{\prime}, y}=\lambda \eta_{x, y}+\beta \eta_{x^{\prime}, y}$ and hence $\mu(A): H \rightarrow H$ is a linear map. The continuity follows from the estimate $\left|\eta_{x, y}\right| \leq\|\mathbf{A}\|_{\mathcal{M}_{r}\left(\ell^{2}(H)\right)}\|x\|\|y\|$. To show that it is a regular measure, select $\left\{x_{n}: n \in \mathbb{N}\right\}$ dense in $H$. Hence, for any $S \in \mathcal{B}(H)$ we have

$$
\|S\|=\sup \left\{\left\langle S\left(x_{n}\right), x_{m}\right\rangle: n, m \in \mathbb{N}\right\} .
$$

Denoting by $\eta_{n, m}=\eta_{x_{n}, x_{m}}$ we have that for each $B \in \mathfrak{B}(\mathbb{T})$, given $(n, m) \in \mathbb{N} \times \mathbb{N}$ and $\varepsilon>0$, there exists $K_{n, m} \subset B \subset O_{n, m}$ which are compact and open respectively so that

$$
\left|\eta_{n, m}\right|\left(O_{n, m} \backslash K_{n, m}\right)<\varepsilon
$$

Now selecting $K=\overline{\bigcup_{n, m} K_{n, m}}$ and $O=\left(\bigcap_{n, m} O_{n, m}\right)^{\circ}$ we conclude that

$$
\|\mu\|(O \backslash K)<\varepsilon \text {. }
$$

This shows that $\mu \in \mathfrak{M}(\mathbb{T}, \mathcal{B}(H))$.

Using now that

$$
\left\langle T_{\mu}(\phi)(x), y\right\rangle=T_{\eta_{x, y}}(\phi)
$$

for each $\phi \in C(\mathbb{T})$, where $T_{\eta_{x, y}} \in \mathcal{L}(C(\mathbb{T}), \mathbb{C})$ denotes the operator associated to $\eta_{x, y} \in$ $M(\mathbb{T})$, we clearly have that $T_{k j}=\widehat{\mu}(j-k)$ for all $j, k \in \mathbb{N}$.

Select $y_{k}=y \beta_{k}$ for some $\beta_{k} \in \mathbb{C}$ and $\|y\|=1$. From Corollary 5.6 we obtain that

$$
\begin{aligned}
& \int_{0}^{2 \pi}\left\|\left(\sum_{k=1}^{M} \sum_{j=1}^{N} \widehat{\mu}(j-k)\left(x_{j}\right) \beta_{k} \bar{\varphi}_{j}(t) \varphi_{k}(t)\right) \otimes y\right\|_{H \widehat{\otimes} H} \frac{d t}{2 \pi} \\
= & \int_{0}^{2 \pi}\left\|\sum_{l=-M}^{N} \widehat{\mu}(l)\left(\sum_{k=1}^{M} x_{k+l} \beta_{k}\right) \varphi_{-l}(t)\right\| \frac{d t}{2 \pi} \\
\leq & \|\mathbf{A}\|_{\mathcal{M}_{r}\left(\ell^{2}(H)\right)}\|\mathbf{x}\|_{\ell^{2}(H)}\left(\sum_{k=1}^{M}\left|\beta_{k}\right|^{2}\right)^{1 / 2} .
\end{aligned}
$$

Taking $x_{j}=x \alpha_{j}$ such that $\|x\|=1$, we get

$$
\begin{aligned}
& \int_{0}^{2 \pi}\left\|\sum_{l=-M}^{N} \widehat{\mu}(l)(x)\left(\sum_{j-k=l} \alpha_{j} \bar{\varphi}_{j}(t) \beta_{k} \varphi_{k}(t)\right)\right\| \frac{d t}{2 \pi} \\
\leq & \|\mathbf{A}\|_{\mathcal{M}_{r}\left(\ell^{2}(H)\right)}\left(\sum_{j=1}^{N}\left|\alpha_{j}\right|^{2}\right)^{1 / 2}\left(\sum_{k=1}^{M}\left|\beta_{k}\right|^{2}\right)^{1 / 2} .
\end{aligned}
$$


Using now

$$
\gamma(s)=\sum_{l=-M}^{N}\left(\sum_{j-k=l} \beta_{k} \alpha_{j}\right) \varphi_{l}(s) .
$$

Now recall that $\widehat{\mu}(l)(x)=\widehat{\mu}_{x}(l)$ and

$$
\sum_{l=-M}^{N} \widehat{\mu}_{x}(l)\left(\sum_{j-k=l} \alpha_{j} \bar{\varphi}_{j}(t) \beta_{k} \varphi_{k}(t)\right)=\int_{0}^{2 \pi}\left(\sum_{l=-M}^{N} \widehat{\mu}_{x}(l) \varphi_{l}(s)\right) \gamma(-t-s) \frac{d s}{2 \pi} .
$$

Therefore, if $\alpha=\sum_{j=1}^{\infty} \alpha_{j} \varphi_{j}$ and $\beta=\sum_{k=1}^{\infty} \beta_{k} \varphi_{k}$ belong to $L^{2}(\mathbb{T})$, we have that $\gamma(t)=$ $\alpha(t) \beta(-t)$ and

$$
\int_{0}^{2 \pi}\left\|\mu_{x} * \gamma(-t)\right\| \frac{d t}{2 \pi} \leq\|\mathbf{A}\|_{\mathcal{M}_{r}\left(\ell^{2}(H)\right)}\|\alpha\|_{L^{2}(\mathbb{T})}\|\beta\|_{L^{2}(\mathbb{T})} .
$$

To show that $\mu_{x} \in M(\mathbb{T}, H)$, due to Lemma 3.8 , it suffices to prove that

$$
\sup _{0<r<1}\left\|\mu_{x} * P_{r}\right\|_{L^{1}(\mathbb{T}, H)}<\infty .
$$

Choosing $\beta(t)=\alpha(t)=\sqrt{1-r^{2}} /\left|1-r e^{i t}\right|$ we obtain that $\gamma(t)=P_{r}(t)$ and from 5.2 we get (5.3) and the estimate $\left\|\mu_{x}\right\|_{M(\mathbb{T}, H)} \leq\|\mathbf{A}\|_{\mathcal{M}_{r}\left(\ell^{2}(H)\right)}$. This finishes the proof.

\section{References}

[1] A. B. Aleksandrov and V. V. Peller, Hankel and Toeplitz-Schur multipliers, Math. Ann. 324 (2002), no. 2, 277-327.

[2] G. Bennett, Schur multipliers, Duke Math. J. 44 (1977), no. 3, 603-639.

[3] O. Blasco, Fourier analysis for vector-measures on compact abelian groups, Rev. R. Acad. Cienc. Exactas Fís. Nat. Ser. A Math. RACSAM 110 (2016), no. 2, 519-539.

[4] J. Diestel, J. H. Fourie and J. Swart, The Metric Theory of Tensor Products, Grothendieck's Résumé Revisited, American Mathematical Society, Providence, RI, 2008.

[5] J. Diestel, H. Jarchow and A. Tonge, Absolutely Summing Operators, Cambridge Studies in Advanced Mathematics 43, Cambridge University Press, Cambridge, 1995.

[6] J. Diestel and J. J. Uhl, Vector Measures, Mathematical Surveys 15, American Mathematical Society, Providence, RI, 1977.

[7] N. Dinculeanu, Vector Measures, VEB Deutscher Verlag der Wissenschaften, Berlin, 1966. 
[8] W. Hengsen, A simpler proof of Singer's representation theorem, Proc. Amer. Math. Soc. 124 (1996), no. 10, 3211-3212.

[9] T. Hytönen, J. van Neerven, M. Veraar and L. Weis, Analysis in Banach Spaces I: Martingales and Littlewood-Paley Theory, A Series of Modern Surveys in Mathematics 63, Springer, Cham, 2016.

[10] L.-E. Persson and N. Popa, Matrix Spaces and Schur Multipliers, Matriceal Harmonic Analysis, World Scientific, Hackensack, NJ, 2014.

[11] R. A. Ryan, Introduction to Tensor Products of Banach Spaces, Springer Monographs in Mathematics, Springer-Verlag London, London, 2002.

[12] J. Schur, Bemerkungen zur Theorie der beschränkten Bilinearformen mit unendlich vielen Veränderlichen, J. Reine Angew. Math. 140 (1911), 1-28.

[13] I. Singer, Linear functionals on the space of continuous mappings of a compact Hausdorf space into a Banach space (in Russian), Rev. Roum. Math. Pures Appl. 2 (1957), 301-315.

[14] I. Singer, Sur les applications linéaires intégrales des espaces de fonctions continues I, Rev. Math. Pures Appl. 4 (1959), 391-401.

[15] O. Toeplitz, Zur Theorie der quadratischen und bilinearen Formen von unendlichvielen Veränderlichen, Math. Ann. 70 (1911), no. 3, 351-376.

Oscar Blasco and Ismael García-Bayona

Departamento de Análisis Matemático, Universidad de Valencia, 46100 Burjassot, Valencia, Spain

E-mail address: oscar.blasco@uv.es, garbais@uv.es 